Geometry $\&$ Topology

Volume 9 (2005) 1115-1146

Published: 1 June 2005

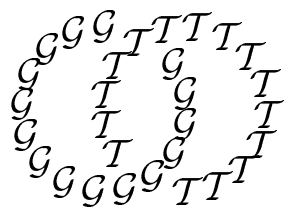

\title{
Deformations of asymptotically cylindrical coassociative submanifolds with fixed boundary
}

\author{
DOMINic JoYCE \\ SEMA SALUR \\ Lincoln College, Oxford, OX1 3DR, UK \\ and \\ Department of Mathematics, Northwestern University, IL 60208, USA \\ Email: dominic.joyce@lincoln.oxford.ac.uk, salur@math.northwestern.edu
}

\begin{abstract}
McLean proved that the moduli space of coassociative deformations of a compact coassociative 4 -submanifold $C$ in a $G_{2}$-manifold $(M, \varphi, g)$ is a smooth manifold of dimension equal to $b_{+}^{2}(C)$. In this paper, we show that the moduli space of coassociative deformations of a noncompact, asymptotically cylindrical coassociative 4 -fold $C$ in an asymptotically cylindrical $G_{2}$-manifold $(M, \varphi, g)$ is also a smooth manifold. Its dimension is the dimension of the positive subspace of the image of $H_{\mathrm{cs}}^{2}(C, \mathbb{R})$ in $H^{2}(C, \mathbb{R})$.
\end{abstract}

AMS Classification numbers Primary: 53C38, 53C15, 53C21

Secondary: $58 \mathrm{~J} 05$

Keywords: Calibrated geometries, asymptotically cylindrical manifolds, $G_{2}-$ manifolds, coassociative submanifolds, elliptic operators.

Proposed: Rob Kirby

Seconded: Simon Donaldson, Gang Tian
Received: 12 August 2004

Accepted: 7 May 2005 


\section{Introduction}

Let $(M, g)$ be a Riemannian 7 -manifold whose holonomy group $\operatorname{Hol}(g)$ is the exceptional holonomy group $G_{2}$ (or, more generally, a subgroup of $G_{2}$ ). Then $M$ is naturally equipped with a constant 3 -form $\varphi$ and 4 -form $* \varphi$. We call $(M, \varphi, g)$ a $G_{2}$-manifold. Complete examples of Riemannian 7-manifolds with holonomy $G_{2}$ were constructed by Bryant and Salamon [3], and compact examples by Joyce [7] and Kovalev [11].

Now $\varphi$ and $* \varphi$ are calibrations on $M$, in the sense of Harvey and Lawson [5]. The corresponding calibrated submanifolds in $M$ are called associative 3 folds and coassociative 4-folds, respectively. They are distinguished classes of minimal 3- and 4-submanifolds in $(M, g)$ with a rich structure, that can be thought of as analogous to complex curves and surfaces in a Calabi-Yau 3-fold.

Harvey and Lawson [5] introduced four types of calibrated geometries. Special Lagrangian submanifolds of Calabi-Yau manifolds, associative and coassociative submanifolds of $G_{2}$ manifolds and Cayley submanifolds of Spin(7) manifolds. Calibrated geometries have been of growing interest over the past few years and represent one of the most mysterious classes of minimal submanifolds 12, 13. A great deal of progress has been made recently in the field of special Lagrangian submanifolds that arise in mirror symmetry for Calabi-Yau manifolds and plays a significant role in string theory, for references see [8]. As one might expect, another promising direction for future investigation is calibrated submanifolds in $G_{2}$ and Spin(7) manifolds. Recently, some progress has been made in constructing such submanifolds [6, 17, 18, and in understanding their deformations [1, 14].

The deformation theory of compact calibrated submanifolds was studied by McLean [22]. He showed that if $C$ is a compact coassociative 4 -fold in a $G_{2}-$ manifold $(M, \varphi, g)$, then the moduli space $\mathcal{M}_{C}$ of coassociative deformations of $C$ is smooth, with dimension $b_{+}^{2}(C)$.

This paper proves an analogue of McLean's theorem for a special class of noncompact coassociative 4-folds. The situation we are interested in is when $(M, \varphi, g)$ is an asymptotically cylindrical $G_{2}$-manifold, that is, it is a noncompact 7-manifold with one end asymptotic to the cylinder $X \times \mathbb{R}$ on a CalabiYau 3 -fold $X$. The natural class of noncompact coassociative 4 -folds in $M$ are asymptotically cylindrical coassociative 4 -folds $C$, asymptotic at infinity in $M$ to a cylinder $L \times \mathbb{R}$, where $L$ is a special Lagrangian 3 -fold in $X$, with phase $i$. Understanding the deformations of such submanifolds when the ambient $G_{2}$-manifold decomposes into connected sum of two pieces will provide the 
necessary technical framework towards completing the Floer homology program for coassociative submanifolds, [13.

In particular, we prove the following theorem.

Theorem 1.1 Let $(M, \varphi, g)$ be a $G_{2}$-manifold asymptotic to $X \times(R, \infty)$ with decay rate $\alpha<0$, where $X$ is a Calabi-Yau 3-fold. Let $C$ be a coassociative 4-fold in $M$ asymptotic to $L \times\left(R^{\prime}, \infty\right)$ for $R^{\prime}>R$ with decay rate $\beta$ for $\alpha \leqslant \beta<0$, where $L$ is a special Lagrangian 3 -fold in $X$ with phase $i$.

If $\gamma<0$ is small enough then the moduli space $\mathcal{M}_{C}^{\gamma}$ of asymptotically cylindrical coassociative submanifolds in $M$ close to $C$, and asymptotic to $L \times\left(R^{\prime}, \infty\right)$ with decay rate $\gamma$, is a smooth manifold of dimension $\operatorname{dim} V_{+}$, where $V_{+}$is the positive subspace of the image of $H_{\mathrm{cs}}^{2}(C, \mathbb{R})$ in $H^{2}(C, \mathbb{R})$.

The principal analytic tool we shall use to prove this is the theory of weighted Sobolev spaces on manifolds with ends, developed by Lockhart and McOwen [15. 16]. The important fact is that elliptic partial differential operators on exterior forms such as $\mathrm{d}+\mathrm{d}^{*}$ or $\mathrm{d}^{*} \mathrm{~d}+\mathrm{dd}^{*}$ on the noncompact 4-manifold $C$ are Fredholm operators between appropriate Banach spaces of forms, and we can describe their kernels and cokernels.

Results similar to Theorem 1.1 on the deformations of classes of noncompact special Lagrangian $m$-folds were proved by Marshall [19] and Pacini [24] for asymptotically conical special Lagrangian $m$-folds, and by Joyce [7, 9] for special Lagrangian $m$-folds with isolated conical singularities. Marshall and Joyce also use the Lockhart-McOwen framework, but Pacini uses a different analytical approach due to Melrose 20, 21. Note also that Kovalev [1] constructs compact $G_{2}$-manifolds by gluing together two noncompact, asymptotically cylindrical $G_{2}-$ manifolds.

We begin in Section 2 with an introduction to $G_{2}$-manifolds and coassociative submanifolds, including a sketch of the proof of McLean's theorem on deformations of compact coassociative 4-folds, and the definitions of asymptotically cylindrical $G_{2}$-manifolds and coassociative 4 -folds. Section 3 introduces the weighted Sobolev spaces of Lockhart and McOwen, and determines the kernel and cokernel of the elliptic operator $\mathrm{d}_{+}+\mathrm{d}^{*}$ on $C$ used in the proof. Finally, Section 4 proves Theorem 1.1. using Banach space techniques and elliptic regularity.

Remark 1.2 In [1], Kovalev constructs asymptotically cylindrical manifolds $X$ with holonomy $S U(3)$. Then $X \times \mathcal{S}^{1}$ is an asymptotically cylindrical $G_{2}-$ manifold, though with holonomy $S U(3)$ rather than $G_{2}$. One can find examples of asymptotically cylindrical coassociative 4 -folds $C$ in $X \times \mathcal{S}^{1}$ of two types: 
(a) $C=C^{\prime} \times \mathrm{pt}$, for $C^{\prime}$ an asymptotically cylindrical complex surface in $X$; or

(b) $C=L \times \mathcal{S}^{1}$, for $L$ an asymptotically cylindrical special Lagrangian 3-fold in $X$, with phase $i$.

Examples of type (a) can be constructed using algebraic geometry: if $X=\bar{X} \backslash D$ for $\bar{X}$ a Fano 3-fold and $D$ a smooth divisor in $\bar{X}$, then we can take $C=\bar{C} \backslash D$ for $\bar{C}$ a smooth divisor in $\bar{X}$ intersecting $D$ transversely. Examples of type (b) can be found by choosing the Calabi-Yau 3-fold $(X, J, \omega, \Omega)$ to have an antiholomorphic involution $\sigma: X \rightarrow X$ with $\sigma^{*}(J)=-J, \sigma^{*}(\omega)=-\omega$ and $\sigma^{*}(\Omega)=-\bar{\Omega}$. Then the fixed points $L$ of $\sigma$ are a special Lagrangian 3 -fold with phase $i$, and each infinite end of $L$ is asymptotically cylindrical.

We can then apply Theorem 1.1 to these examples. One can show that if $\widetilde{C}$ is a small deformation of a coassociative 4 -fold $C$ of type (a) or (b) then $\widetilde{C}$ is also of type (a) or (b) and thus, Theorem 1.1 implies analogous results on the deformation theory of asymptotically cylindrical complex surfaces and special Lagrangian 3-folds in asymptotically cylindrical Calabi-Yau 3-folds.

\section{Introduction to $G_{2}$ geometry}

We now give background material on $G_{2}$-manifolds and their coassociative submanifolds that will be needed later. A good reference on $G_{2}$ geometry is Joyce [7, Sections 10-12], and a good reference on calibrated geometry is Harvey and Lawson [5].

\section{1 $G_{2}-$ manifolds and coassociative submanifolds}

Let $\left(x_{1}, \ldots, x_{7}\right)$ be coordinates on $\mathbb{R}^{7}$. Write $\mathrm{d} \mathbf{x}_{i j \ldots l}$ for the exterior form $\mathrm{d} x_{i} \wedge \mathrm{d} x_{j} \wedge \cdots \wedge \mathrm{d} x_{l}$ on $\mathbb{R}^{7}$. Define a metric $g_{0}$, a 3 -form $\varphi_{0}$ and a 4 -form $* \varphi_{0}$ on $\mathbb{R}^{7}$ by $g_{0}=\mathrm{d} x_{1}^{2}+\cdots+\mathrm{d} x_{7}^{2}$,

$$
\begin{aligned}
\varphi_{0} & =\mathrm{d} \mathbf{x}_{123}+\mathrm{d} \mathbf{x}_{145}+\mathrm{d} \mathbf{x}_{167}+\mathrm{d}_{246}-\mathrm{d} \mathbf{x}_{257}-\mathrm{d} \mathbf{x}_{347}-\mathrm{d} \mathbf{x}_{356} \text { and } \\
* \varphi_{0} & =\mathrm{d} \mathbf{x}_{4567}+\mathrm{d} \mathbf{x}_{2367}+\mathrm{d} \mathbf{x}_{2345}+\mathrm{d} \mathbf{x}_{1357}-\mathrm{d} \mathbf{x}_{1346}-\mathrm{d} \mathbf{x}_{1256}-\mathrm{d} \mathbf{x}_{1247} .
\end{aligned}
$$

The subgroup of $G L(7, \mathbb{R})$ preserving $\varphi_{0}$ is the exceptional Lie group $G_{2}$. It also preserves $g_{0}, * \varphi_{0}$ and the orientation on $\mathbb{R}^{7}$. It is a compact, semisimple, 14-dimensional Lie group, a subgroup of $\mathrm{SO}(7)$.

A $G_{2}$-structure on a 7 -manifold $M$ is a principal subbundle of the frame bundle of $M$, with structure group $G_{2}$. Each $G_{2}$-structure gives rise to a 3-form $\varphi$ and 
a metric $g$ on $M$, such that every tangent space of $M$ admits an isomorphism with $\mathbb{R}^{7}$ identifying $\varphi$ and $g$ with $\varphi_{0}$ and $g_{0}$ respectively. By an abuse of notation, we will refer to $(\varphi, g)$ as a $G_{2}$-structure.

Proposition 2.1 Let $M$ be a 7 -manifold and $(\varphi, g)$ a $G_{2}$-structure on $M$. Then the following are equivalent:

(i) $\operatorname{Hol}(g) \subseteq G_{2}$, and $\varphi$ is the induced 3 -form,

(ii) $\nabla \varphi=0$ on $M$, where $\nabla$ is the Levi-Civita connection of $g$, and

(iii) $\mathrm{d} \varphi=\mathrm{d}^{*} \varphi=0$ on $M$.

We call $\nabla \varphi$ the torsion of the $G_{2}$-structure $(\varphi, g)$, and when $\nabla \varphi=0$ the $G_{2}$-structure is torsion-free. A triple $(M, \varphi, g)$ is called a $G_{2}-$ manifold if $M$ is a 7-manifold and $(\varphi, g)$ a torsion-free $G_{2}$-structure on $M$. If $g$ has holonomy $\operatorname{Hol}(g) \subseteq G_{2}$, then $g$ is Ricci-flat. For explicit, complete examples of $G_{2}-$ manifolds see Bryant and Salamon [3], and for compact examples see Joyce [7] and Kovalev [11. Here are the basic definitions in calibrated geometry, due to Harvey and Lawson [5].

Definition 2.2 Let $(M, g)$ be a Riemannian manifold. An oriented tangent $k$-plane $V$ on $M$ is a vector subspace $V$ of some tangent space $T_{x} M$ to $M$ with $\operatorname{dim} V=k$, equipped with an orientation. If $V$ is an oriented tangent $k$-plane on $M$ then $\left.g\right|_{V}$ is a Euclidean metric on $V$, so combining $\left.g\right|_{V}$ with the orientation on $V$ gives a natural volume form $\operatorname{vol}_{V}$ on $V$, which is a $k$-form on $V$.

Now let $\varphi$ be a closed $k$-form on $M$. We say that $\varphi$ is a calibration on $M$ if for every oriented $k$-plane $V$ on $M$ we have $\left.\varphi\right|_{V} \leqslant \operatorname{vol}_{V}$. Here $\left.\varphi\right|_{V}=\alpha \cdot \operatorname{vol}_{V}$ for some $\alpha \in \mathbb{R}$, and $\left.\varphi\right|_{V} \leqslant \operatorname{vol}_{V}$ if $\alpha \leqslant 1$. Let $N$ be an oriented submanifold of $M$ with dimension $k$. Then each tangent space $T_{x} N$ for $x \in N$ is an oriented tangent $k$-plane. We call $N$ a calibrated submanifold if $\left.\varphi\right|_{T_{x} N}=\operatorname{vol}_{T_{x} N}$ for all $x \in N$.

Calibrated submanifolds are automatically minimal submanifolds (see [5. Theorem II.4.2]). There are two natural classes of calibrated submanifolds in $G_{2}-$ manifolds.

Definition 2.3 Let $(M, \varphi, g)$ be a $G_{2}$-manifold, as above. Then the 3 -form $\varphi$ is a calibration on $(M, g)$. We define an associative 3 -fold in $M$ to be a 3-submanifold of $M$ calibrated with respect to $\varphi$. Similarly, the Hodge star $* \varphi$ of $\varphi$ is a calibration 4 -form on $(M, g)$. We define a coassociative 4 -fold in $M$ to be a 4 -submanifold of $M$ calibrated with respect to $* \varphi$. 
McLean [22, Prop. 4.4] gives an alternative description of coassociative 4-folds:

Proposition 2.4 Let $(M, \varphi, g)$ be a $G_{2}$-manifold, and $C$ a 4 -dimensional submanifold of $M$. Then $C$ admits an orientation making it into a coassociative 4 -fold if and only if $\left.\varphi\right|_{C} \equiv 0$.

\subsection{Deformations of compact coassociative $4-$ folds}

Here is the main result in the deformation theory of coassociative 4-folds, proved by McLean [22, Theorem 4.5]. As our sign conventions for $\varphi_{0}, * \varphi_{0}$ in (11) are different to McLean's, we use self-dual 2-forms in place of McLean's anti-selfdual 2-forms.

Theorem 2.5 Let $(M, \varphi, g)$ be a $G_{2}$-manifold, and $C$ a compact coassociative 4-fold in $M$. Then the moduli space $\mathcal{M}_{C}$ of coassociative 4-folds isotopic to $C$ in $M$ is a smooth manifold of dimension $b_{+}^{2}(C)$.

Sketch proof Suppose for simplicity that $C$ is an embedded submanifold. There is a natural orthogonal decomposition $\left.T M\right|_{C}=T C \oplus \nu$, where $\nu \rightarrow C$ is the normal bundle of $C$ in $M$. There is a natural isomorphism $\nu \cong \Lambda_{+}^{2} T^{*} C$, constructed as follows. Let $x \in C$ and $V \in \nu_{x}$. Then $V$ lies in $T_{x} M$, so $\left.V \cdot \varphi\right|_{x} \in$ $\Lambda^{2} T_{x}^{*} M$, and $\left.\left(\left.V \cdot \varphi\right|_{x}\right)\right|_{T_{x} C} \in \Lambda^{2} T_{x}^{*} C$. Moreover $\left.\left(\left.V \cdot \varphi\right|_{x}\right)\right|_{T_{x} C}$ actually lies in $\Lambda_{+}^{2} T_{x}^{*} C$, the bundle of self-dual 2 -forms on $C$, and the map $\left.V \mapsto\left(\left.V \cdot \varphi\right|_{x}\right)\right|_{T_{x} C}$ defines an isomorphism $\nu \stackrel{\cong}{\longrightarrow} \Lambda_{+}^{2} T^{*} C$.

For small $\epsilon>0$, write $B_{\epsilon}(\nu)$ for the subbundle of $\nu$ with fibre at $x$ the open ball about 0 in $\left.\nu\right|_{x}$ with radius $\epsilon$. Then the exponential map exp: $\nu \rightarrow M$ induces a diffeomorphism between $B_{\epsilon}(\nu)$ and a small tubular neighbourhood $T_{C}$ of $C$ in $M$. The isomorphism $\nu \cong \Lambda_{+}^{2} T^{*} C$ gives a diffeomorphism exp: $B_{\epsilon}\left(\Lambda_{+}^{2} T^{*} C\right) \rightarrow$ $T_{C}$. Let $\pi: T_{C} \rightarrow C$ be the obvious projection.

Under this identification, submanifolds $\widetilde{C}$ in $T_{C} \subset M$ which are $C^{1}$ close to $C$ are identified with the graphs $\Gamma_{\zeta_{+}^{2}}$ of small smooth sections $\zeta_{+}^{2}$ of $\Lambda_{+}^{2} T^{*} C$ lying in $B_{\epsilon}\left(\Lambda_{+}^{2} T^{*} C\right)$. For each $\zeta_{+}^{2} \in C^{\infty}\left(B_{\epsilon}\left(\Lambda_{+}^{2} T^{*} C\right)\right)$ the graph $\Gamma_{\zeta_{+}^{2}}$ is a $4-$ submanifold of $B_{\epsilon}\left(\Lambda_{+}^{2} T^{*} C\right)$, and so $\widetilde{C}=\exp \left(\Gamma_{\zeta_{+}^{2}}\right)$ is a 4 -submanifold of $T_{C}$. We need to know: which 2 -forms $\zeta_{+}^{2}$ correspond to coassociative 4 -folds $\widetilde{C}$ in $T_{C}$ ?

$\widetilde{C}$ is coassociative if $\left.\varphi\right|_{\widetilde{C}} \equiv 0$. Now $\left.\pi\right|_{\widetilde{C}}: \widetilde{C} \rightarrow C$ is a diffeomorphism, so we can push $\left.\varphi\right|_{\widetilde{C}}$ down to $C$, and regard it as a function of $\zeta_{+}^{2}$. That is, we define

$$
Q: C^{\infty}\left(B_{\epsilon}\left(\Lambda_{+}^{2} T^{*} C\right)\right) \rightarrow C^{\infty}\left(\Lambda^{3} T^{*} C\right) \text { by } Q\left(\zeta_{+}^{2}\right)=\pi_{*}\left(\left.\varphi\right|_{\exp \left(\Gamma_{\zeta_{+}^{2}}\right)}\right) .
$$


Then the moduli space $\mathcal{M}_{C}$ is locally isomorphic near $C$ to the set of small self-dual $2-$ forms $\zeta_{+}^{2}$ on $C$ with $\left.\varphi\right|_{\exp \left(\Gamma_{\zeta_{+}^{2}}\right)} \equiv 0$, that is, to a neighborhood of 0 in $Q^{-1}(0)$.

To understand the equation $Q\left(\zeta_{+}^{2}\right)=0$, note that at $x \in C,\left.Q\left(\zeta_{+}^{2}\right)\right|_{x}$ depends on the tangent space to $\Gamma_{\zeta_{+}^{2}}$ at $\left.\zeta_{+}^{2}\right|_{x}$, and so on $\left.\zeta_{+}^{2}\right|_{x}$ and $\left.\nabla \zeta_{+}^{2}\right|_{x}$. Thus the functional form of $Q$ is

$$
\left.Q\left(\zeta_{+}^{2}\right)\right|_{x}=F\left(x,\left.\zeta_{+}^{2}\right|_{x},\left.\nabla \zeta_{+}^{2}\right|_{x}\right) \text { for } x \in C,
$$

where $F$ is a smooth function of its arguments. Hence $Q\left(\zeta_{+}^{2}\right)=0$ is a nonlinear first order $P D E$ in $\zeta_{+}^{2}$. As $\varphi$ is closed, $\left.\varphi\right|_{C} \equiv 0$, and $\Gamma_{\zeta_{+}^{2}}$ is isotopic to $C$, we see that $\left.\varphi\right|_{\Gamma_{+}^{2}}$ is an exact 3 -form on $\Gamma_{\zeta_{+}^{2}}$, so that $Q\left(\zeta_{+}^{2}\right)$ is exact. The linearization $\mathrm{d} Q(0)$ of $Q$ at $\zeta_{+}^{2}=0$ is

$$
\mathrm{d} Q(0)(\beta)=\lim _{\epsilon \rightarrow 0}\left(\epsilon^{-1} Q(\epsilon \beta)\right)=\mathrm{d} \beta .
$$

Therefore $\operatorname{Ker}(\mathrm{d} Q(0))$ is the vector space $\mathcal{H}_{+}^{2}$ of closed self-dual 2 -forms $\beta$ on $C$, which by Hodge theory is a finite-dimensional vector space isomorphic to $H_{+}^{2}(C, \mathbb{R})$, with dimension $b_{+}^{2}(C)$. This is the Zariski tangent space of $\mathcal{M}_{C}$ at $C$, the infinitesimal deformation space of $C$ as a coassociative 4 -fold.

To complete the proof we must show that $\mathcal{M}_{C}$ is locally isomorphic to its Zariski tangent space $\mathcal{H}_{+}^{2}$, and so is a smooth manifold of dimension $b_{+}^{2}(C)$. To do this rigorously requires some technical analytic machinery, which is passed over in a few lines in [22, p. 731]. Here is one way to do it.

As $Q$ maps from $\Lambda_{+}^{2} T^{*} C$ with fibre $\mathbb{R}^{3}$ to $\Lambda^{3} T^{*} C$ with fibre $\mathbb{R}^{4}$, it is overdetermined, and not elliptic. To turn it into an elliptic operator, define

$$
\begin{gathered}
P: C^{\infty}\left(B_{\epsilon}\left(\Lambda_{+}^{2} T^{*} C\right)\right) \times C^{\infty}\left(\Lambda^{4} T^{*} C\right) \rightarrow C^{\infty}\left(\Lambda^{3} T^{*} C\right) \\
\text { by } P\left(\zeta_{+}^{2}, \zeta^{4}\right)=Q\left(\zeta_{+}^{2}\right)+\mathrm{d}^{*} \zeta^{4} .
\end{gathered}
$$

Then the linearization of $P$ at $(0,0)$ is

$$
\mathrm{d} P(0,0):\left(\zeta_{+}^{2}, \zeta^{4}\right) \mapsto \mathrm{d} \zeta_{+}^{2}+\mathrm{d}^{*} \zeta^{4},
$$

which is elliptic. Since ellipticity is an open condition, $P$ is elliptic near $(0,0)$ in $C^{\infty}\left(B_{\epsilon}\left(\Lambda_{+}^{2} T^{*} C\right)\right) \times C^{\infty}\left(\Lambda^{4} T^{*} C\right)$.

Suppose $P\left(\zeta_{+}^{2}, \zeta^{4}\right)=0$. Then $Q\left(\zeta_{+}^{2}\right)=-\mathrm{d}^{*} \zeta^{4}$, so

$$
\left\|\mathrm{d}^{*} \zeta^{4}\right\|_{L^{2}}^{2}=-\left\langle\mathrm{d}^{*} \zeta^{4}, Q\left(\zeta_{+}^{2}\right)\right\rangle_{L^{2}}=-\left\langle\zeta^{4}, \mathrm{~d}\left(Q\left(\zeta_{+}^{2}\right)\right)\right\rangle_{L^{2}}=0
$$

by integration by parts, since $Q\left(\zeta_{+}^{2}\right)$ is exact. Hence $P\left(\zeta_{+}^{2}, \zeta^{4}\right)=0$ if and only if $Q\left(\zeta_{+}^{2}\right)=\mathrm{d}^{*} \zeta^{4}=0$. But 4 -forms with $\mathrm{d}^{*} \zeta^{4}=0$ are constant, and the vector space of such $\zeta^{4}$ is $H^{4}(C, \mathbb{R})$. Thus, $P^{-1}(0)=Q^{-1}(0) \times H^{4}(C, \mathbb{R})$. 
Because $C^{\infty}\left(\Lambda_{+}^{2} T^{*} C\right), C^{\infty}\left(\Lambda^{3} T^{*} C\right)$ are not Banach spaces, we extend $P$ in (2) to act on Sobolev spaces $L_{l+2}^{p}\left(\Lambda_{+}^{2} T^{*} C\right), L_{l+2}^{p}\left(\Lambda^{3} T^{*} C\right)$ for $p>4$ and $l \geqslant 0$, giving

$$
\begin{aligned}
& \widetilde{P}: L_{l+2}^{p}\left(B_{\epsilon}\left(\Lambda_{+}^{2} T^{*} C\right)\right) \times L_{l+2}^{p}\left(\Lambda^{4} T^{*} C\right) \rightarrow L_{l+1}^{p}\left(\Lambda^{3} T^{*} C\right), \\
& \widetilde{P}:\left(\zeta_{+}^{2}, \zeta^{4}\right) \mapsto \pi_{*}\left(\left.\varphi\right|_{\Gamma_{\zeta_{+}^{2}}}\right)+\mathrm{d}^{*} \zeta^{4} .
\end{aligned}
$$

Then $\widetilde{P}$ is a smooth map of Banach manifolds.

Let $\mathcal{H}^{3}$ be the vector space of closed and coclosed 3 -forms on $C$, so that $\mathcal{H}^{3} \cong$ $H^{3}(C, \mathbb{R})$ by Hodge theory, and $V_{l+1}^{p}$ be the Banach subspace of $L_{l+1}^{p}\left(\Lambda^{3} T^{*} C\right)$ $L^{2}$-orthogonal to $\mathcal{H}^{3}$. Then one can show that $\widetilde{P}$ maps into $V_{l+1}^{p}$, and the linearization

$$
\begin{aligned}
& \mathrm{d} \widetilde{P}(0,0): L_{l+2}^{p}\left(\Lambda_{+}^{2} T^{*} C\right) \times L_{l+2}^{p}\left(\Lambda^{4} T^{*} C\right) \rightarrow V_{l+1}^{p}, \\
& \mathrm{~d} \widetilde{P}(0,0):\left(\zeta_{+}^{2}, \zeta^{4}\right) \mapsto \mathrm{d} \zeta_{+}^{2}+\mathrm{d}^{*} \zeta^{4}
\end{aligned}
$$

is then surjective as a map of Banach spaces.

Thus, $\widetilde{P}: L_{l+2}^{p}\left(B_{\epsilon}\left(\Lambda_{+}^{2} T^{*} C\right)\right) \times L_{l+2}^{p}\left(\Lambda^{4} T^{*} C\right) \rightarrow V_{l+1}^{p}$ is a smooth map of Banach manifolds, with $\mathrm{d} \widetilde{P}(0,0)$ surjective. The Implicit Mapping Theorem for Banach spaces (Theorem 4.4) now implies that $\widetilde{P}^{-1}(0)$ is near 0 a smooth submanifold, locally isomorphic to $\operatorname{Ker}(\mathrm{d} \widetilde{P}(0))$. But $\widetilde{P}\left(\zeta_{+}^{2}, \zeta^{4}\right)=0$ is an elliptic equation for small $\zeta_{+}^{2}, \zeta^{4}$, and so elliptic regularity implies that solutions $\left(\zeta_{+}^{2}, \zeta^{4}\right)$ are smooth.

Therefore $\widetilde{P}^{-1}(0)=P^{-1}(0)$ near 0 , and also $\operatorname{Ker}(\mathrm{d} \widetilde{P}(0,0))=\operatorname{Ker}(\mathrm{d} P(0,0))$. Hence $P^{-1}(0)$ is, near $(0,0)$, a smooth manifold locally isomorphic to the kernel $\operatorname{Ker}(\mathrm{d} P(0,0))$. So from above $Q^{-1}(0)$ is near 0 a smooth manifold locally isomorphic to $\operatorname{Ker}(\mathrm{d} Q(0))$. Thus, $\mathcal{M}_{C}$ is near $C$ a smooth manifold locally isomorphic to $H_{+}^{2}(C, \mathbb{R})$. This completes the proof.

\subsection{Asymptotically cylindrical $G_{2}$-manifolds and coassociative 4 -folds}

We first define cylindrical and asymptotically cylindrical $G_{2}-$ manifolds.

Definition 2.6 A $G_{2}$-manifold $\left(M_{0}, \varphi_{0}, g_{0}\right)$ is called cylindrical if $M_{0}=X \times \mathbb{R}$ and $\left(\varphi_{0}, g_{0}\right)$ is compatible with this product structure, that is,

$$
\varphi_{0}=\operatorname{Re} \Omega+\omega \wedge \mathrm{d} t \quad \text { and } \quad g_{0}=g_{X}+\mathrm{d} t^{2},
$$

where $X$ is a (connected, compact) Calabi-Yau 3-fold with Kähler form $\omega$, Riemannian metric $g_{X}$ and holomorphic (3,0)-form $\Omega$. 
Definition 2.7 A connected, complete $G_{2}$-manifold $(M, \varphi, g)$ is called asymptotically cylindrical with decay rate $\alpha$ if there exists a cylindrical $G_{2}-$ manifold $\left(M_{0}, \varphi_{0}, g_{0}\right)$ with $M_{0}=X \times \mathbb{R}$ as above, a compact subset $K \subset M$, a real number $R$, and a diffeomorphism $\Psi: X \times(R, \infty) \rightarrow M \backslash K$ such that $\Psi^{*}(\varphi)=\varphi_{0}+\mathrm{d} \xi$ for some smooth 2 -form $\xi$ on $X \times(R, \infty)$ with $\left|\nabla^{k} \xi\right|=O\left(e^{\alpha t}\right)$ on $X \times(R, \infty)$ for all $k \geqslant 0$, where $\nabla$ is the Levi-Civita connection of the cylindrical metric $g_{0}$.

The point of this is that $M$ has one end modelled on $X \times(R, \infty)$, and as $t \rightarrow \infty$ in $(R, \infty)$ the $G_{2}$-structure $(\varphi, g)$ on $M$ converges to order $O\left(e^{\alpha t}\right)$ to the cylindrical $G_{2}$-structure on $X \times(R, \infty)$, with all of its derivatives. We suppose $M$ and $X$ are connected, that is, we allow $M$ to have only one end.

This is because one can use Cheeger-Gromoll splitting theorem [4] to show that an orientable, connected, asymptotically cylindrical Riemannian manifold with Ricci-flat metric $g$ can have at most 2 cylindrical ends. In the case when there are 2 cylindrical ends then there is reduction in the holonomy group $\operatorname{Hol}(g)$ and $(M, g)$ is a cylinder. One can also show that reduction in holonomy can be obtained by just using the analytic set-up for Fredholm properties of an elliptic operator on noncompact manifolds, 25].

Here are the analogous definitions for coassociative submanifolds.

Definition 2.8 Let $\left(M_{0}, \varphi_{0}, g_{0}\right)$ and $X$ be as in Definition 2.6. A submanifold $C_{0}$ of $M_{0}$ is called cylindrical if $C_{0}=L \times \mathbb{R}$ for some compact submanifold $L$ in $X$, not necessarily connected. $C_{0}$ is coassociative if and only if $L$ is a special Lagrangian 3 -fold with phase $i$ in the Calabi-Yau 3-fold $X$.

Definition 2.9 Let $\left(M_{0}, \varphi_{0}, g_{0}\right), X,(M, \varphi, g), K, \Psi$ and $\alpha$ be as in Definitions [2.6] and [2.7] and let $C_{0}=L \times \mathbb{R}$ be a cylindrical coassociative 4 -fold in $M_{0}$, as in Definition 2.8.

A connected, complete coassociative 4 -fold $C$ in $(M, \varphi, g)$ is called asymptotically cylindrical with decay rate $\beta$ for $\alpha \leqslant \beta<0$ if there exists a compact subset $K^{\prime} \subset C$, a normal vector field $v$ on $L \times\left(R^{\prime}, \infty\right)$ for some $R^{\prime}>R$, and a diffeomorphism $\Phi: L \times\left(R^{\prime}, \infty\right) \rightarrow C \backslash K^{\prime}$ such that the diagram

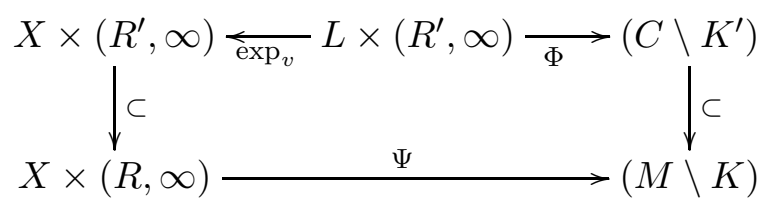

commutes, and $\left|\nabla^{k} v\right|=O\left(e^{\beta t}\right)$ on $L \times\left(R^{\prime}, \infty\right)$ for all $k \geqslant 0$. 
Here we require $C$ but not $L$ to be connected, that is, we allow $C$ to have multiple ends. The point of Definition 2.9 is to find a good way to say that a submanifold $C$ in $M$ is asymptotic to the cylinder $C_{0}$ in $M_{0}=X \times \mathbb{R}$ as $t \rightarrow \infty$ in $\mathbb{R}$, to order $O\left(e^{\beta t}\right)$. We do this by writing $C$ near infinity as the graph of a normal vector field $v$ to $C_{0}=L \times \mathbb{R}$ in $M_{0}=X \times \mathbb{R}$, and requiring $v$ and its derivatives to be $O\left(e^{\beta t}\right)$.

\section{Infinitesimal deformations of $C$}

Let $(M, \varphi, g)$ be an asymptotically cylindrical $G_{2}$-manifold asymptotic to $X \times$ $(R, \infty)$, and $C$ an asymptotically cylindrical coassociative 4 -fold in $M$ asymptotic to $L \times\left(R^{\prime}, \infty\right)$. We wish to study the moduli space $\mathcal{M}_{C}^{\gamma}$ of asymptotically

cylindrical deformations $\widetilde{C}$ of $C$ in $M$ with rate $\gamma$. To do this we modify the proof of Theorem 2.5 in Section 2 for the case when $C$ is compact. There we modelled $\mathcal{M}_{C}$ on $\widetilde{P}^{-1}(0)$ for a nonlinear map $\widetilde{P}$ between Banach spaces, whose linearization $\mathrm{d} \widetilde{P}(0,0)$ at 0 was the Fredholm map between Sobolev spaces

$$
\mathrm{d}_{+}+\mathrm{d}^{*}: L_{l+2}^{p}\left(\Lambda_{+}^{2} T^{*} C\right) \times L_{l+2}^{p}\left(\Lambda^{4} T^{*} C\right) \longrightarrow L_{l+1}^{p}\left(\Lambda^{3} T^{*} C\right) .
$$

Now when $C$ is not compact, as in the asymptotically cylindrical case, (4) is not in general Fredholm, and the proof of Theorem [2.5 fails. To repair it we use the analytical framework for asymptotically cylindrical manifolds developed by Lockhart and McOwen in [15, 16, involving weighted Sobolev spaces $L_{k, \gamma}^{p}\left(\Lambda^{r} T^{*} C\right)$. Roughly speaking, elements of $L_{k, \gamma}^{p}\left(\Lambda^{r} T^{*} C\right)$ are $L_{k}^{p} r$-forms on $C$ which decay like $O\left(e^{\gamma t}\right)$ on the end $L \times\left(R^{\prime}, \infty\right)$. This has the advantage of building the decay rate $\gamma$ into the proof from the outset.

This section will study the weighted analogue of (4),

$$
\mathrm{d}_{+}+\mathrm{d}^{*}: L_{l+2, \gamma}^{p}\left(\Lambda_{+}^{2} T^{*} C\right) \times L_{l+2, \gamma}^{p}\left(\Lambda^{4} T^{*} C\right) \longrightarrow L_{l+1, \gamma}^{p}\left(\Lambda^{3} T^{*} C\right),
$$

for small $\gamma<0$. It will be shown in Section 4 to be the linearization at 0 of a nonlinear operator $P$ for which $\mathcal{M}_{C}^{\gamma}$ is locally modelled on $P^{-1}(0)$.

Section 3.1 introduces weighted Sobolev spaces, and the Lockhart-McOwen theory of elliptic operators between them. Then Sections 3.2 and 3.3 compute the kernel and cokernel of (5) for small $\gamma<0$, and Section 3.4 determines the set of rates $\gamma$ for which (5) is Fredholm. 


\subsection{Elliptic operators on asymptotically cylindrical manifolds}

We now sketch parts of the theory of analysis on manifolds with cylindrical ends due to Lockhart and McOwen [15, 16]. We begin with some elementary definitions.

Definition 3.1 Let $(C, g)$ be an asymptotically cylindrical Riemannian manifold. That is, there is a Riemannian cylinder $\left(L \times \mathbb{R}, g_{0}\right)$ with $L$ compact, a compact subset $K^{\prime} \subset C$ and a diffeomorphism $\Phi: C \backslash K^{\prime} \rightarrow L \times\left(R^{\prime}, \infty\right)$ such that

$$
\nabla_{0}^{k}\left(\Phi_{*}(g)-g_{0}\right)=O\left(e^{\beta t}\right) \quad \text { for all } k \geqslant 0
$$

for some rate $\beta<0$, where $\nabla_{0}$ is the Levi-Civita connection of $g_{0}$ on $L \times \mathbb{R}$.

Let $E_{0}$ be a cylindrical vector bundle on $L \times \mathbb{R}$, that is, a vector bundle on $L \times \mathbb{R}$ invariant under translations in $\mathbb{R}$. Let $h_{0}$ be a smooth family of metrics on the fibres of $E_{0}$ and $\nabla_{E_{0}}$ a connection on $E_{0}$ preserving $h_{0}$, with $h_{0}, \nabla_{E_{0}}$ invariant under translations in $\mathbb{R}$.

Let $E$ be a vector bundle on $C$ equipped with metrics $h$ on the fibres, and a connection $\nabla_{E}$ on $E$ preserving $h$. We say that $E, h, \nabla_{E}$ are asymptotic to $E_{0}, h_{0}, \nabla_{E_{0}}$ if there exists an identification $\Phi_{*}(E) \cong E_{0}$ on $L \times\left(R^{\prime}, \infty\right)$ such that $\Phi_{*}(h)=h_{0}+O\left(e^{\beta t}\right)$ and $\Phi_{*}\left(\nabla_{E}\right)=\nabla_{E_{0}}+O\left(e^{\beta t}\right)$ as $t \rightarrow \infty$. Then we call $E, h, \nabla_{E}$ asymptotically cylindrical.

Choose a smooth function $\rho: C \rightarrow \mathbb{R}$ such that $\Phi^{*}(\rho) \equiv t$ on $L \times\left(R^{\prime}, \infty\right)$. This prescribes $\rho$ on $C \backslash K^{\prime}$, so we only have to extend $\rho$ over the compact set $K^{\prime}$. For $p \geq 1, k \geq 0$ and $\gamma \in \mathbb{R}$ we define the weighted Sobolev space $L_{k, \gamma}^{p}(E)$ to be the set of sections $s$ of $E$ that are locally integrable and $k$ times weakly differentiable and for which the norm

$$
\|s\|_{L_{k, \gamma}^{p}}=\left(\sum_{j=0}^{k} \int_{C} e^{-\gamma \rho}\left|\nabla_{E}^{j} s\right|^{p} \mathrm{~d} V\right)^{1 / p}
$$

is finite. Then $L_{k, \gamma}^{p}(E)$ is a Banach space. Since $\rho$ is uniquely determined except on the compact set $K^{\prime}$, different choices of $\rho$ give the same space $L_{k, \gamma}^{p}(E)$, with equivalent norms.

For instance, the $r$-forms $E=\Lambda^{r} T^{*} C$ on $C$ with metric $g$ and the LeviCivita connection are automatically asymptotically cylindrical, and if $C$ is an oriented 4-manifold then the self-dual 2-forms $\Lambda_{+}^{2} T^{*} C$ are also asymptotically cylindrical. We consider partial differential operators on asymptotically cylindrical manifolds. 
Definition 3.2 In the situation of Definition 3.1 suppose $E, F$ are two asymptotically cylindrical vector bundles on $C$, asymptotic to cylindrical vector bundles $E_{0}, F_{0}$ on $L \times \mathbb{R}$. Let $A_{0}: C^{\infty}\left(E_{0}\right) \rightarrow C^{\infty}\left(F_{0}\right)$ be a linear partial differential operator of order $k$ which is cylindrical, that is, invariant under translations in $\mathbb{R}$.

Suppose $A: C^{\infty}(E) \rightarrow C^{\infty}(F)$ is a linear partial differential operator of order $k$ on $C$. We say that $A$ is asymptotic to $A_{0}$ if under the identifications $\Phi_{*}(E) \cong$ $E_{0}, \Phi_{*}(F) \cong F_{0}$ on $L \times\left(R^{\prime}, \infty\right)$ we have $\Phi_{*}(A)=A_{0}+O\left(e^{\beta t}\right)$ as $t \rightarrow \infty$ for $\beta<0$. Then we call $A$ an asymptotically cylindrical operator. It is easy to show that $A$ extends to bounded linear operators

$$
A_{k+l, \gamma}^{p}: L_{k+l, \gamma}^{p}(E) \longrightarrow L_{l, \gamma}^{p}(F)
$$

for all $p>1, l \geqslant 0$ and $\gamma \in \mathbb{R}$.

Now suppose $A$ is an elliptic operator. (7) is Fredholm if and only if $\gamma$ does not lie in a discrete set $\mathcal{D}_{A_{0}} \subset \mathbb{R}$, which we now define.

Definition 3.3 In Definition 3.2 suppose $A$ and $A_{0}$ are elliptic operators on $C$ and $L \times \mathbb{R}$, so that $E, F$ have the same fibre dimensions. Extend $A_{0}$ to the complexifications $A_{0}: C^{\infty}\left(E_{0} \otimes_{\mathbb{R}} \mathbb{C}\right) \rightarrow C^{\infty}\left(F_{0} \otimes_{\mathbb{R}} \mathbb{C}\right)$. Define $\mathcal{D}_{A_{0}}$ to be the set of $\gamma \in \mathbb{R}$ such that for some $\delta \in \mathbb{R}$ there exists a nonzero section $s \in C^{\infty}\left(E_{0} \otimes_{\mathbb{R}} \mathbb{C}\right)$ invariant under translations in $\mathbb{R}$ such that $A_{0}\left(e^{(\gamma+i \delta) t} s\right)=0$.

Then Lockhart and McOwen prove [16, Theorem 1.1]:

Theorem 3.4 Let $(C, g)$ be a Riemannian manifold asymptotic to $\left(L \times \mathbb{R}, g_{0}\right)$, and $A: C^{\infty}(E) \rightarrow C^{\infty}(F)$ an elliptic partial differential operator on $C$ of order $k$ between vector bundles $E, F$ on $C$, asymptotic to the cylindrical elliptic operator $A_{0}: C^{\infty}\left(E_{0}\right) \rightarrow C^{\infty}\left(F_{0}\right)$ on $L \times \mathbb{R}$. Define $\mathcal{D}_{A_{0}}$ as above.

Then $\mathcal{D}_{A_{0}}$ is a discrete subset of $\mathbb{R}$, and for $p>1, l \geqslant 0$ and $\gamma \in \mathbb{R}$, the extension $A_{k+l, \gamma}^{p}: L_{k+l, \gamma}^{p}(E) \rightarrow L_{l, \gamma}^{p}(F)$ is Fredholm if and only if $\gamma \notin \mathcal{D}_{A_{0}}$.

Suppose $\gamma \notin \mathcal{D}_{A_{0}}$. Then $A_{k+l, \gamma}^{p}$ is Fredholm, so its kernel $\operatorname{Ker}\left(A_{k+l, \gamma}^{p}\right)$ is finitedimensional. Let $e \in \operatorname{Ker}\left(A_{k+l, \gamma}^{p}\right)$. Then by an elliptic regularity result [15, Theorem 3.7.2] we have $e \in L_{k+m, \gamma}^{p}(E)$ for all $m \geqslant 0$. The weighted Sobolev Embedding Theorem [15, Theorem 3.10] then implies that $e \in L_{k+m, \delta}^{r}(E)$ for all $r>1, m \geqslant 0$ and $\delta>\gamma$, and $e$ is smooth. But $\operatorname{Ker}\left(A_{k+1, \gamma}^{p}\right)$ is invariant under small changes of $\gamma$ in $\mathbb{R} \backslash \mathcal{D}_{A_{0}}$, so $e \in L_{k+m, \gamma}^{r}(E)$ for all $r>1$ and $m \geqslant 0$. This proves: 
Proposition 3.5 For $\gamma \notin \mathcal{D}_{A_{0}}$ the kernel $\operatorname{Ker}\left(A_{k+l, \gamma}^{p}\right)$ is independent of $p, l$, and is a finite-dimensional vector space of smooth sections of $E$.

When $\gamma \notin \mathcal{D}_{A_{0}}$, as $A_{k+l, \gamma}^{p}$ is Fredholm the cokernel

$$
\operatorname{Coker}\left(A_{k+l, \gamma}^{p}\right)=L_{l, \gamma}^{p}(F) / A_{k+l, \gamma}^{p}\left(L_{k+l, \gamma}^{p}(E)\right)
$$

of $A_{k+l, \gamma}^{p}$ is also finite-dimensional. To understand it, consider the formal adjoint $A^{*}: C^{\infty}(F) \rightarrow C^{\infty}(E)$ of $A$. This is also an asymptotically cylindrical linear elliptic partial differential operator of order $k$ on $C$, with the property that

$$
\langle A e, f\rangle_{L^{2}(F)}=\left\langle e, A^{*} f\right\rangle_{L^{2}(E)}
$$

for compactly-supported $e \in C^{\infty}(E)$ and $f \in C^{\infty}(F)$.

Then for $p>1, l \geqslant 0$ and $\gamma \notin \mathcal{D}_{A_{0}}$, the dual operator of (7) is

$$
\left(A^{*}\right)_{-l,-\gamma}^{q}: L_{-l,-\gamma}^{q}(F) \longrightarrow L_{-k-l,-\gamma}^{q}(E)
$$

where $q>1$ is defined by $\frac{1}{p}+\frac{1}{q}=1$. Here we mean that $L_{-k-l,-\gamma}^{q}(E)$, $L_{-l,-\gamma}^{q}(F)$ are isomorphic to the Banach space duals of $L_{k+l, \gamma}^{p}(E), L_{l, \gamma}^{p}(F)$, and these isomorphisms identify $\left(A^{*}\right)_{-l,-\gamma}^{q}$ with the dual linear map to (7).

Now there is a problem with (8), as it involves Sobolev spaces with negative numbers of derivatives $-l,-k-l$. Such Sobolev spaces exist as spaces of distributions. But we can avoid defining or using these spaces, by the following trick. We are interested in $\operatorname{Ker}\left(\left(A^{*}\right)_{-l,-\gamma}^{q}\right)$, as it is dual to $\operatorname{Coker}\left(A_{k+l, \gamma}^{p}\right)$. The elliptic regularity argument above showing $\operatorname{Ker}\left(A_{k+l, \gamma}^{p}\right)$ is independent of $l$ also holds for negative differentiability, so we have $\operatorname{Ker}\left(\left(A^{*}\right)_{-l,-\gamma}^{q}\right)=\operatorname{Ker}\left(\left(A^{*}\right)_{k+m,-\gamma}^{q}\right)$ for $m \in \mathbb{Z}$, and in particular for $m \geqslant 0$. So we deduce:

Proposition 3.6 In Theorem 3.4, let $A^{*}$ be the formal adjoint of $A$. Then for all $\gamma \notin \mathcal{D}_{A_{0}}, p, q>1$ with $\frac{1}{p}+\frac{1}{q}=1$ and $l, m \geqslant 0$ there is a natural isomorphism

$$
\operatorname{Coker}\left(A_{k+l, \gamma}^{p}\right) \cong \operatorname{Ker}\left(\left(A^{*}\right)_{k+m,-\gamma}^{q}\right)^{*}
$$

When $\gamma \notin \mathcal{D}_{A_{0}}$ we see from (9) that the index of $A_{k+l, \gamma}^{p}$ is

$$
\operatorname{ind}\left(A_{k+l, \gamma}^{p}\right)=\operatorname{dim} \operatorname{Ker}\left(A_{k+l, \gamma}^{p}\right)-\operatorname{dim} \operatorname{Ker}\left(\left(A^{*}\right)_{k+m,-\gamma}^{q}\right) \text {. }
$$

Lockhart and McOwen show [16, Theorem 6.2] that for $\gamma, \delta \in \mathbb{R} \backslash \mathcal{D}_{A_{0}}$ with $\gamma \leqslant \delta$ we have

$$
\operatorname{ind}\left(A_{k+l, \delta}^{p}\right)-\operatorname{ind}\left(A_{k+l, \gamma}^{p}\right)=\sum_{\epsilon \in \mathcal{D}} d(\epsilon),
$$


where $d(\epsilon) \geqslant 1$ is the dimension of the a vector space of solutions $s \in C^{\infty}\left(E_{0} \otimes_{\mathbb{R}}\right.$ $\mathbb{C})$ of a prescribed form with $A_{0}(s)=0$.

\section{$3.2 \quad d+d^{*}$ and $d^{*} d+d d^{*}$ on an asymptotically cylindrical manifold}

Let $(C, g)$ be an oriented asymptotically cylindrical Riemannian $n$-manifold asymptotic to a Riemannian cylinder $\left(L \times \mathbb{R}, g_{0}\right)$, where $g_{0}=g_{L}+\mathrm{d} t^{2}$ and $\left(L, g_{L}\right)$ is a compact oriented Riemannian $(n-1)$-manifold. Consider the asymptotically cylindrical linear elliptic operators

$$
\mathrm{d}+\mathrm{d}^{*} \text { and } \mathrm{d}^{*} \mathrm{~d}+\mathrm{dd}^{*}: \bigoplus_{k=0}^{n} C^{\infty}\left(\Lambda^{k} T^{*} C\right) \longrightarrow \bigoplus_{k=0}^{n} C^{\infty}\left(\Lambda^{k} T^{*} C\right) .
$$

We shall apply the theory of Section 3.1 to study the extensions

$$
\begin{aligned}
\left(\mathrm{d}+\mathrm{d}^{*}\right)_{l+2, \gamma}^{p}: & \bigoplus_{k=0}^{n} L_{l+2, \gamma}^{p}\left(\Lambda^{k} T^{*} C\right) \\
\left(\mathrm{d}^{*} \mathrm{~d}+\mathrm{dd}^{*}\right)_{l+2, \gamma}^{p}: \bigoplus_{k=0}^{n} L_{l+2, \gamma}^{p}\left(\Lambda^{k} T^{*} C\right) & \longrightarrow \bigoplus_{k=0}^{n} L_{l+1, \gamma}^{p}\left(\Lambda^{k} T^{*} C\right),
\end{aligned}
$$

for $p>1, l \geqslant 0$ and $\gamma \in \mathbb{R}$, and their kernels and cokernels.

Lemma 3.7 We have $\operatorname{Ker}\left(\left(\mathrm{d}+\mathrm{d}^{*}\right)_{l+2, \gamma}^{p}\right) \subseteq \operatorname{Ker}\left(\left(\mathrm{d}^{*} \mathrm{~d}+\mathrm{dd}^{*}\right)_{l+2, \gamma}^{p}\right)$ for all $p>1$, $l \geqslant 0$ and $\gamma \in \mathbb{R}$, and equality holds if $\gamma<0$.

Proof Since $\mathrm{d}^{*} \mathrm{~d}+\mathrm{dd}^{*}=\left(\mathrm{d}+\mathrm{d}^{*}\right)^{2}$ we have $\operatorname{Ker}\left(\mathrm{d}+\mathrm{d}^{*}\right) \subseteq \operatorname{Ker}\left(\mathrm{d}^{*} \mathrm{~d}+\mathrm{dd}^{*}\right)$ on any space of twice differentiable forms, giving the inclusion. Suppose $\gamma<0$ and $\chi \in \operatorname{Ker}\left(\left(\mathrm{d}^{*} \mathrm{~d}+\mathrm{dd}^{*}\right)_{l+2, \gamma}^{p}\right)$. Write $\chi=\sum_{k=0}^{n} \chi_{k}$ for $\chi_{k} \in L_{l+2, \gamma}^{p}\left(\Lambda^{k} T^{*} C\right)$. Then $\chi_{k} \in \operatorname{Ker}\left(\left(\mathrm{d}^{*} \mathrm{~d}+\mathrm{dd}^{*}\right)_{l+2, \gamma}^{p}\right)$, as $\mathrm{d}^{*} \mathrm{~d}+\mathrm{dd}^{*}$ takes $k$-forms to $k$-forms.

If $\gamma<0$ then each $\chi_{k}$ lies in $L_{2}^{2}\left(\Lambda^{k} T^{*} C\right)$, and

$\left\|\mathrm{d} \chi_{k}\right\|_{L^{2}}^{2}+\left\|\mathrm{d}^{*} \chi_{k}\right\|_{L^{2}}^{2}=\left\langle\mathrm{d} \chi_{k}, \mathrm{~d} \chi_{k}\right\rangle_{L^{2}}+\left\langle\mathrm{d}^{*} \chi_{k}, \mathrm{~d}^{*} \chi_{k}\right\rangle_{L^{2}}=\left\langle\chi_{k},\left(\mathrm{~d}^{*} \mathrm{~d}+\mathrm{dd}^{*}\right) \chi_{k}\right\rangle_{L^{2}}=0$.

Thus $\mathrm{d}^{*} \chi_{k}=\mathrm{d} \chi_{k}=0$, so that $\chi_{k}$ and hence $\chi$ lies in $\operatorname{Ker}\left(\left(\mathrm{d}+\mathrm{d}^{*}\right)_{l+2, \gamma}^{p}\right)$.

For $|\gamma|$ close to zero we can say more about the kernels of (13) and (14).

Proposition 3.8 Suppose $p, q>1, l, m \geqslant 0$ and $\gamma<0$ with $\frac{1}{p}+\frac{1}{q}=1$ and $[\gamma,-\gamma] \cap \mathcal{D}_{\left(\mathrm{d}+\mathrm{d}^{*}\right)_{0}}=[\gamma,-\gamma] \cap \mathcal{D}_{\left(\mathrm{d}^{*} \mathrm{~d}+\mathrm{dd}^{*}\right)_{0}}=\{0\}$. Then

$$
\begin{aligned}
\operatorname{Ker}\left(\left(\mathrm{d}+\mathrm{d}^{*}\right)_{l+2, \gamma}^{p}\right) & =\operatorname{Ker}\left(\left(\mathrm{d}^{*} \mathrm{~d}+\mathrm{dd}^{*}\right)_{l+2, \gamma}^{p}\right), \\
\operatorname{Ker}\left(\left(\mathrm{d}+\mathrm{d}^{*}\right)_{m+2,-\gamma}^{q}\right) & =\operatorname{Ker}\left(\left(\mathrm{d}^{*} \mathrm{~d}+\mathrm{dd}^{*}\right)_{m+2,-\gamma}^{q}\right), \quad \text { and } \\
\operatorname{dim} \operatorname{Ker}\left(\left(\mathrm{d}+\mathrm{d}^{*}\right)_{m+2,-\gamma}^{q}\right) & =\operatorname{dim} \operatorname{Ker}\left(\left(\mathrm{d}+\mathrm{d}^{*}\right)_{l+2, \gamma}^{p}\right)+\sum_{k=0}^{n-1} b^{k}(L) .
\end{aligned}
$$

Moreover all four kernels consist of smooth closed and coclosed forms. 
Proof As $[\gamma,-\gamma] \cap \mathcal{D}_{\left(\mathrm{d}+\mathrm{d}^{*}\right)_{0}}=\{0\}$

$$
\operatorname{ind}\left(\left(\mathrm{d}+\mathrm{d}^{*}\right)_{m+2,-\gamma}^{q}\right)-\operatorname{ind}\left(\left(\mathrm{d}+\mathrm{d}^{*}\right)_{l+2, \gamma}^{p}\right)=2 \sum_{k=0}^{n-1} b^{k}(L) \text {. }
$$

This is because from (11), the l.h.s. of (18) is the dimension of the solution space of $\left(\mathrm{d}+\mathrm{d}^{*}\right)_{0} \chi=0$ on $L \times \mathbb{R}$ for $\chi$ independent of $t \in \mathbb{R}$. The space of such $\chi$ is the direct sum over $k=0, \ldots, n-1$ of the spaces of $k$-forms $\eta$ and $(k+1)$-forms $\eta \wedge \mathrm{d} t$ for $\eta \in C^{\infty}\left(\Lambda^{k} T^{*} L\right)$ with $\mathrm{d} \eta=\mathrm{d}^{*} \eta=0$. By Hodge theory we deduce (18).

Now $\mathrm{d}+\mathrm{d}^{*}$ is formally self adjoint, that is, $A^{*}=A$ in the notation of Section 3.1 Thus

$$
\begin{gathered}
\operatorname{ind}\left(\left(\mathrm{d}+\mathrm{d}^{*}\right)_{m+2,-\gamma}^{q}\right)=-\operatorname{ind}\left(\left(\mathrm{d}+\mathrm{d}^{*}\right)_{l+2, \gamma}^{p}\right)= \\
\operatorname{dim} \operatorname{Ker}\left(\left(\mathrm{d}+\mathrm{d}^{*}\right)_{m+2,-\gamma}^{q}\right)-\operatorname{dim} \operatorname{Ker}\left(\left(\mathrm{d}+\mathrm{d}^{*}\right)_{l+2, \gamma}^{p}\right)
\end{gathered}
$$

by (10), and equation (17) follows from (18). As $[\gamma,-\gamma] \cap \mathcal{D}_{\left(\mathrm{d}^{*} \mathrm{~d}+\mathrm{dd}^{*}\right)_{0}}=\{0\}$, the same proof shows that

$$
\operatorname{dim} \operatorname{Ker}\left(\left(\mathrm{d}^{*} \mathrm{~d}+\mathrm{dd}^{*}\right)_{m+2,-\gamma}^{q}\right)=\operatorname{dim} \operatorname{Ker}\left(\left(\mathrm{d}^{*} \mathrm{~d}+\mathrm{dd}^{*}\right)_{l+2, \gamma}^{p}\right)+\sum_{k=0}^{n-1} b^{k}(L),
$$

since the solutions of $\left(\mathrm{d}^{*} \mathrm{~d}+\mathrm{dd}^{*}\right)_{0} \chi=0$ and $\left(\mathrm{d}+\mathrm{d}^{*}\right)_{0} \chi=0$ for $\chi$ on $L \times \mathbb{R}$ independent of $t$ coincide. Lemma 3.7 proves (15), and combining this with (17) and (19) yields

$$
\operatorname{dim} \operatorname{Ker}\left(\left(\mathrm{d}+\mathrm{d}^{*}\right)_{m+2,-\gamma}^{q}\right)=\operatorname{dim} \operatorname{Ker}\left(\left(\mathrm{d}^{*} \mathrm{~d}+\mathrm{dd}^{*}\right)_{m+2,-\gamma}^{q}\right) .
$$

As the right hand side of (16) contains the left by Lemma 3.7, this implies (16).

It remains to show the four kernels consist of smooth closed and coclosed forms. Let $\chi$ lie in one of the kernels, and write $\chi=\sum_{k=0}^{n} \chi_{k}$ for $\chi_{k}$ a $k$-form. Since $\left(\mathrm{d}^{*} \mathrm{~d}+\mathrm{dd}^{*}\right) \chi=0$ we have $\left(\mathrm{d}^{*} \mathrm{~d}+\mathrm{dd}^{*}\right) \chi_{k}=0$, as $\mathrm{d}^{*} \mathrm{~d}+\mathrm{dd}^{*}$ takes $k$-forms to $k$-forms. Thus $\chi_{k}$ lies in the same kernel, so $\left(\mathrm{d}+\mathrm{d}^{*}\right) \chi_{k}=0$ by (15) or (16). But $\mathrm{d} \chi_{k}$ and $\mathrm{d}^{*} \chi_{k}$ lie in different vector spaces, so $\mathrm{d} \chi_{k}=\mathrm{d}^{*} \chi_{k}=0$ for all $k$. Hence $\mathrm{d} \chi=\mathrm{d}^{*} \chi=0$, and $\chi$ is closed and coclosed. Smoothness follows by elliptic regularity.

As the forms $\chi$ in $\operatorname{Ker}\left(\left(\mathrm{d}+\mathrm{d}^{*}\right)_{l+2, \gamma}^{p}\right)$ are closed we can map them to de Rham cohomology $H^{*}(C, \mathbb{R})$ by $\chi \mapsto[\chi]$. We identify the kernel and image of this map.

Proposition 3.9 Suppose $p>1, l \geqslant 0$ and $\gamma<0$ with $[\gamma,-\gamma] \cap \mathcal{D}_{\left(\mathrm{d}+\mathrm{d}^{*}\right)_{0}}=$ $[\gamma,-\gamma] \cap \mathcal{D}_{\left(\mathrm{d}^{*} \mathrm{~d}+\mathrm{dd}^{*}\right)_{0}}=\{0\}$. Then the map $\operatorname{Ker}\left(\left(\mathrm{d}+\mathrm{d}^{*}\right)_{l+2, \gamma}^{p}\right) \rightarrow H^{*}(C, \mathbb{R})$ given by $\chi \mapsto[\chi]$ is injective, with image that of the natural map $H_{\mathrm{cs}}^{*}(C, \mathbb{R}) \rightarrow$ $H^{*}(C, \mathbb{R})$. 
Proof Lockhart [15, Ex. 0.14] shows that the vector space $\mathcal{H}^{2}\left(\Lambda^{k} T^{*} C, g\right)$ of closed, coclosed $k$-forms in $L^{2}\left(\Lambda^{k} T^{*} C\right)$ on an asymptotically cylindrical Riemannian manifold $(C, g)$ is isomorphic under $\chi \mapsto[\chi]$ with the image of $H_{\mathrm{cs}}^{k}(C, \mathbb{R})$ in $H^{k}(C, \mathbb{R})$. Taking the direct sum over $k=0, \ldots, n$, this implies that for $l \geqslant 0$ the map

$$
\operatorname{Ker}\left(\left(\mathrm{d}+\mathrm{d}^{*}\right)_{l+2,0}^{2}\right) \rightarrow H^{*}(C, \mathbb{R}), \quad \chi \mapsto[\chi]
$$

is injective, with image that of the natural map $H_{\mathrm{cs}}^{*}(C, \mathbb{R}) \rightarrow H^{*}(C, \mathbb{R})$.

Using Proposition [3.5. $\gamma \notin \mathcal{D}_{\left(\mathrm{d}+\mathrm{d}^{*}\right)_{0}}$ and $\gamma<0$ we have

$$
\operatorname{Ker}\left(\left(\mathrm{d}+\mathrm{d}^{*}\right)_{l+2, \gamma}^{p}\right)=\operatorname{Ker}\left(\left(\mathrm{d}+\mathrm{d}^{*}\right)_{l+2, \gamma}^{2}\right) \subseteq \operatorname{Ker}\left(\left(\mathrm{d}+\mathrm{d}^{*}\right)_{l+2,0}^{2}\right) .
$$

Therefore $\chi \mapsto[\chi]$ is injective on $\operatorname{Ker}\left(\left(\mathrm{d}+\mathrm{d}^{*}\right)_{l+2, \gamma}^{p}\right)$, with image contained in that of $H_{\mathrm{cs}}^{*}(C, \mathbb{R}) \rightarrow H^{*}(C, \mathbb{R})$. It remains to show $\chi \mapsto[\chi]$ is surjective on this image.

Suppose $\eta \in H^{j}(C, \mathbb{R})$ lies in the image of $H_{\mathrm{cS}}^{j}(C, \mathbb{R})$. Then we may write $\eta=[\phi]$ for $\phi$ a smooth, closed, compactly-supported $j$-form on $C$. Hence $\mathrm{d}^{*} \phi \in \bigoplus_{k=0}^{n} L_{l+1, \gamma}^{p}\left(\Lambda^{k} T^{*} C\right)$. We shall show that $\mathrm{d}^{*} \phi$ lies in the image of (14) with $l+1$ in place of $l$. Since $\gamma \notin \mathcal{D}_{\left(\mathrm{d}^{*} \mathrm{~d}+\mathrm{dd}^{*}\right)_{0}}$, as in Section 3.1 this holds if and only if $\left\langle\mathrm{d}^{*} \phi, \xi\right\rangle_{L^{2}}=0$ for all $\xi$ in $\operatorname{Ker}\left(\left(\mathrm{d}^{*} \mathrm{~d}+\mathrm{dd}^{*}\right)_{m+2,-\gamma}^{q}\right)$.

But all such $\xi$ are closed by Proposition 3.8, so $\left\langle\mathrm{d}^{*} \phi, \xi\right\rangle_{L^{2}}=\langle\phi, \mathrm{d} \xi\rangle_{L^{2}}=0$. Therefore $\mathrm{d}^{*} \phi=\left(\mathrm{d}^{*} \mathrm{~d}+\mathrm{dd}^{*}\right) \psi$ for some $\psi \in L_{l+3, \gamma}^{p}\left(\Lambda^{j-1} T^{*} C\right)$. Hence

$$
\left(\mathrm{d}^{*} \mathrm{~d}+\mathrm{dd}^{*}\right)(\phi-\mathrm{d} \psi)=\mathrm{d}\left(\mathrm{d}^{*} \phi-\left(\mathrm{d}^{*} \mathrm{~d}+\mathrm{dd}^{*}\right) \psi\right)=0,
$$

and $\phi-\mathrm{d} \psi$ lies in $\operatorname{Ker}\left(\left(\mathrm{d}^{*} \mathrm{~d}+\mathrm{dd}^{*}\right)_{l+2, \gamma}^{p}\right)$, which is $\operatorname{Ker}\left(\left(\mathrm{d}+\mathrm{d}^{*}\right)_{l+2, \gamma}^{p}\right)$ by (15). As $[\phi-\mathrm{d} \psi]=[\phi]=\eta$ we have proved the surjectivity we need.

\section{$3.3 \mathrm{~d}_{+}+\mathrm{d}^{*}$ on a 4-manifold}

Now we restrict to $\operatorname{dim} C=4$, so that $(C, g)$ is an oriented asymptotically cylindrical Riemannian 4-manifold asymptotic to a Riemannian cylinder $\left(L \times \mathbb{R}, g_{0}\right)$. In Section 4 we will take $C$ to be an asymptotically cylindrical coassociative 4 fold. Consider the asymptotically cylindrical linear elliptic operator

$$
\mathrm{d}_{+}+\mathrm{d}^{*}: C^{\infty}\left(\Lambda_{+}^{2} T^{*} C\right) \oplus C^{\infty}\left(\Lambda^{4} T^{*} C\right) \longrightarrow C^{\infty}\left(\Lambda^{3} T^{*} C\right) .
$$

Here $d_{+}$is the restriction of $d$ to the self-dual 2 -forms. We use this notation to distinguish $\mathrm{d}_{+}+\mathrm{d}^{*}$ from $\mathrm{d}+\mathrm{d}^{*}$ in (12). Roughly speaking, $\mathrm{d}_{+}+\mathrm{d}^{*}$ is a 
quarter of $\mathrm{d}+\mathrm{d}^{*}$ in (12), as it acts on half of the even forms, rather than on all forms. Its formal adjoint is

$$
\mathrm{d}_{+}^{*}+\mathrm{d}: C^{\infty}\left(\Lambda^{3} T^{*} C\right) \longrightarrow C^{\infty}\left(\Lambda_{+}^{2} T^{*} C\right) \oplus C^{\infty}\left(\Lambda^{4} T^{*} C\right)
$$

where $d_{+}^{*}$ is the projection of $d^{*}$ to the self-dual 2 -forms. We shall apply the results of Section 3.2 to study the extension

$$
\left(\mathrm{d}_{+}+\mathrm{d}^{*}\right)_{l+2, \gamma}^{p}: L_{l+2, \gamma}^{p}\left(\Lambda_{+}^{2} T^{*} C\right) \oplus L_{l+2, \gamma}^{p}\left(\Lambda^{4} T^{*} C\right) \longrightarrow L_{l+1, \gamma}^{p}\left(\Lambda^{3} T^{*} C\right),
$$

for $p>1, l \geqslant 0$ and $\gamma \in \mathbb{R}$. We begin with some algebraic topology.

Suppose for simplicity that $C$ has no compact connected components, so that $H^{4}(C, \mathbb{R})=H_{\mathrm{cs}}^{0}(C, \mathbb{R})=0$. Then $L$ is a compact, oriented 3-manifold, and $C$ is the interior $(\bar{C})^{\circ}$ of a compact, oriented 4-manifold $\bar{C}$ with boundary $\partial \bar{C}=L$. Thus we have a long exact sequence in cohomology:

$$
\begin{aligned}
& 0 \longrightarrow H^{0}(C) \longrightarrow H^{0}(L) \longrightarrow H_{\mathrm{cs}}^{1}(C) \longrightarrow H^{1}(C) \longrightarrow H^{1}(L) \longrightarrow H_{\mathrm{cs}}^{2}(C) \\
& 0 \longleftarrow H_{\mathrm{cs}}^{4}(C) \longleftarrow H^{3}(L) \longleftarrow H^{3}(C) \longleftarrow H_{\mathrm{cs}}^{3}(C) \leftarrow H^{2}(L) \longleftarrow H^{2}(C)
\end{aligned}
$$

where $H^{k}(C)=H^{k}(C, \mathbb{R})$ and $H^{k}(L)=H^{k}(L, \mathbb{R})$ are the de Rham cohomology groups, and $H_{\mathrm{cs}}^{k}(C, \mathbb{R})$ is compactly-supported de Rham cohomology. Let $b^{k}(C)$, $b^{k}(L)$ and $b_{\mathrm{cs}}^{k}(C)$ be the corresponding Betti numbers.

By Poincaré duality we have $H^{k}(C) \cong H_{\mathrm{cs}}^{4-k}(C)^{*}$ and $H^{k}(L) \cong H^{3-k}(L)^{*}$, so that $b^{k}(C)=b_{\mathrm{cs}}^{4-k}(C)$ and $b^{k}(L)=b^{3-k}(L)$. Note that (21) is written so that each vertically aligned pair of spaces are dual vector spaces, and each vertically aligned pair of maps are dual linear maps.

Let $V \subseteq H^{2}(C, \mathbb{R})$ be the image of the natural map $H_{\mathrm{cs}}^{2}(C, \mathbb{R}) \rightarrow H^{2}(C, \mathbb{R})$. Taking alternating sums of dimensions in (21) shows that

$$
\begin{aligned}
\operatorname{dim} V & =b_{\mathrm{cs}}^{2}(C)-b^{1}(L)+b^{1}(C)-b_{\mathrm{cs}}^{1}(C)-b^{0}(L)+b^{0}(C) \\
& =b^{0}(C)+b^{1}(C)+b^{2}(C)-b^{3}(C)-b^{0}(L)-b^{1}(L) .
\end{aligned}
$$

Now the cup product $\cup: H_{\mathrm{cs}}^{2}(C, \mathbb{R}) \times H^{2}(C, \mathbb{R}) \rightarrow \mathbb{R}$ restricted to $H_{\mathrm{cs}}^{2}(C, \mathbb{R}) \times V$ is zero on the product of the kernel of $H_{\mathrm{cs}}^{2}(C, \mathbb{R}) \rightarrow H^{2}(C, \mathbb{R})$ with $V$. Hence it pushes forward to a quadratic form $\cup: V \times V \rightarrow \mathbb{R}$, which is symmetric and nondegenerate.

Suppose $V=V_{+} \oplus V_{-}$is a decomposition of $V$ into subspaces with $\cup$ positive definite on $V_{+}$and negative definite on $V_{-}$. Then $\operatorname{dim} V_{+}$and $\operatorname{dim} V_{-}$are topological invariants of $C, L$. That is, they depend only on $C$ as an oriented 4-manifold, and not on the choice of subspaces $V_{ \pm}$.

We now identify the kernel and cokernel of $\left(\mathrm{d}_{+}+\mathrm{d}^{*}\right)_{l+2, \gamma}^{p}$ in (20) for small $\gamma<0$. 
Theorem 3.10 Let $(C, g)$ be an oriented, asymptotically cylindrical Riemannian 4-manifold asymptotic to $\left(L \times \mathbb{R}, g_{0}\right)$, and use the notation of Sections [3.1 3.2. Suppose $\max \left(\mathcal{D}_{\left(\mathrm{d}_{+}+\mathrm{d}^{*}\right)_{0}} \cap(-\infty, 0)\right)<\gamma<0$, and let $p, q>1$ with $\frac{1}{p}+\frac{1}{q}=1$ and $l, m \geqslant 0$. Then from Section 3.1 the operator $\left(\mathrm{d}_{+}+\mathrm{d}^{*}\right)_{l+2, \gamma}^{p}$ of (20) is Fredholm with

$$
\operatorname{Coker}\left(\left(\mathrm{d}_{+}+\mathrm{d}^{*}\right)_{l+2, \gamma}^{p}\right) \cong \operatorname{Ker}\left(\left(\mathrm{d}_{+}^{*}+\mathrm{d}\right)_{m+2,-\gamma}^{q}\right)^{*} .
$$

The kernel $\operatorname{Ker}\left(\left(\mathrm{d}_{+}+\mathrm{d}^{*}\right)_{l+2, \gamma}^{p}\right)$ is a vector space of smooth, closed, self-dual 2 -forms. The map $\operatorname{Ker}\left(\left(\mathrm{d}_{+}+\mathrm{d}^{*}\right)_{l+2, \gamma}^{p}\right) \rightarrow H^{2}(C, \mathbb{R}), \chi \mapsto[\chi]$ induces an isomorphism of $\operatorname{Ker}\left(\left(\mathrm{d}_{+}+\mathrm{d}^{*}\right)_{l+2, \gamma}^{p}\right)$ with a maximal subspace $V_{+}$of the subspace $V \subseteq H^{2}(C, \mathbb{R})$ defined above on which the cup product $\cup: V \times V \rightarrow \mathbb{R}$ is positive definite. Hence

$$
\operatorname{dim} \operatorname{Ker}\left(\left(\mathrm{d}_{+}+\mathrm{d}^{*}\right)_{l+2, \gamma}^{p}\right)=\operatorname{dim} V_{+},
$$

which is a topological invariant of $C, L$ from above. Also, $\operatorname{Ker}\left(\left(\mathrm{d}_{+}^{*}+\mathrm{d}\right)_{m+2,-\gamma}^{q}\right)$ is a vector space of smooth, closed and coclosed 3-forms.

Proof The first part follows immediately from Section [3.1. As $\operatorname{Ker}\left(\left(\mathrm{d}_{+}+\right.\right.$ $\left.\left.\mathrm{d}^{*}\right)_{l+2, \gamma}^{p}\right)$ and $\operatorname{Ker}\left(\left(\mathrm{d}_{+}^{*}+\mathrm{d}\right)_{m+2,-\gamma}^{q}\right)$ depend only on the connected component of $\mathbb{R} \backslash \mathcal{D}_{\left(\mathrm{d}_{+}+\mathrm{d}^{*}\right)_{0}}$ containing $\gamma$, we can make $|\gamma|$ smaller if necessary to ensure that $[\gamma,-\gamma] \cap \mathcal{D}_{\left(\mathrm{d}+\mathrm{d}^{*}\right)_{0}}=[\gamma,-\gamma] \cap \mathcal{D}_{\left(\mathrm{d}^{*} \mathrm{~d}+\mathrm{dd}^{*}\right)_{0}}=\{0\}$, using the notation of Section 3.2

Suppose $\left(\zeta_{+}^{2}, \zeta^{4}\right) \in \operatorname{Ker}\left(\left(\mathrm{d}_{+}+\mathrm{d}^{*}\right)_{l+2, \gamma}^{p}\right)$. Then $\mathrm{d} \zeta_{+}^{2}+\mathrm{d}^{*} \zeta^{4}=0$, so applying $*$ and noting that $* \zeta_{+}^{2}=\zeta_{+}^{2}$ gives $\mathrm{d}^{*} \zeta_{+}^{2}-\mathrm{d}\left(* \zeta^{4}\right)=0$. Hence $\left(\mathrm{d}+\mathrm{d}^{*}\right)\left(-* \zeta^{4}+\zeta_{+}^{2}+\zeta^{4}\right)=$ 0 , that is, the mixed form $-* \zeta^{4}+\zeta_{+}^{2}+\zeta^{4}$ lies in the kernel of (13). Proposition 3.8 now implies that $\zeta_{+}^{2}$ and $\zeta^{4}$ are smooth, closed and coclosed, and therefore $\zeta_{+}^{2}$ and $\zeta^{4}$ also lie in the kernel, and $\operatorname{Ker}\left(\left(\mathrm{d}_{+}+\mathrm{d}^{*}\right)_{l+2, \gamma}^{p}\right) \subseteq \operatorname{Ker}\left(\left(\mathrm{d}+\mathrm{d}^{*}\right)_{l+2, \gamma}^{p}\right)$, where $\left(\mathrm{d}+\mathrm{d}^{*}\right)_{l+2, \gamma}^{p}$ is as in Section 3.2 .

Since $H^{4}(C, \mathbb{R})=0$, injectivity in Proposition 3.9 implies that $\zeta^{4}=0$. Thus $\operatorname{Ker}\left(\left(\mathrm{d}_{+}+\mathrm{d}^{*}\right)_{l+2, \gamma}^{p}\right)$ is a vector space of smooth, closed, self-dual 2-forms, as we have to prove. Write $\mathcal{H}^{2}$ for the space of 2 -forms in $\operatorname{Ker}\left(\left(\mathrm{d}+\mathrm{d}^{*}\right)_{l+2, \gamma}^{p}\right)$. Then by Proposition 3.9 the map $\mathcal{H}^{2} \rightarrow H^{2}(C, \mathbb{R}), \chi \mapsto[\chi]$ is injective with image that of $H_{\mathrm{cs}}^{2}(C, \mathbb{R})$ in $H^{2}(C, \mathbb{R})$, which is $V$ in the notation above.

Under this isomorphism, the cup product on $V$ is given by

$$
\chi \cup \xi=\int_{C} \chi \wedge \xi=\int_{C}(\chi, * \xi) \mathrm{d} V_{g}=\langle\chi, * \xi\rangle_{L^{2}}
$$


for $\chi, \xi \in \mathcal{H}^{2}$. The Hodge star $*$ maps $\mathcal{H}^{2} \cong V$ to itself with $*^{2}=1$. Let $V_{ \pm}$be the \pm 1 eigenspaces of $*$ on $V$. Then $V=V_{+} \oplus V_{-}$, and (24) implies that $U$ is positive definite on $V_{+}$and negative definite on $V_{-}$. Hence $\operatorname{dim} V_{+}$ is a topological invariant, from above. But $\operatorname{Ker}\left(\left(\mathrm{d}_{+}+\mathrm{d}^{*}\right)_{l+2, \gamma}^{p}\right)$ is the self-dual 2 -forms in $\mathcal{H}^{2}$. Thus $\chi \mapsto[\chi]$ induces an isomorphism of $\operatorname{Ker}\left(\left(\mathrm{d}_{+}+\mathrm{d}^{*}\right)_{l+2, \gamma}^{p}\right)$ with $V_{+}$, as we have to prove.

Finally, suppose $\zeta^{3} \in \operatorname{Ker}\left(\left(\mathrm{d}_{+}^{*}+\mathrm{d}\right)_{m+2,-\gamma}^{q}\right)$, so that $\mathrm{d}_{+}^{*} \zeta^{3}=\mathrm{d} \zeta^{3}=0$. Then

$$
0=\mathrm{d}^{*}\left(\mathrm{~d} \zeta^{3}\right)+2 \mathrm{~d}\left(\mathrm{~d}_{+}^{*} \zeta^{3}\right)=\mathrm{d}^{*} \mathrm{~d} \zeta^{3}+\mathrm{d}\left(\mathrm{d}^{*} \zeta^{3}-\mathrm{d}\left(* \zeta^{3}\right)\right)=\left(\mathrm{d}^{*} \mathrm{~d}+\mathrm{dd}^{*}\right) \zeta_{3},
$$

so $\zeta^{3}$ lies in $\operatorname{Ker}\left(\left(\mathrm{d}^{*} \mathrm{~d}+\mathrm{dd}^{*}\right)_{m+2,-\gamma}^{q}\right)$, which consists of smooth closed and coclosed forms by Proposition 3.8. Thus $\operatorname{Ker}\left(\left(\mathrm{d}_{+}^{*}+\mathrm{d}\right)_{m+2,-\gamma}^{q}\right)$ is a vector space of smooth, closed and coclosed 3-forms.

We can say more about the cokernel (22). Its dimension is

$$
\operatorname{dim} \operatorname{Ker}\left(\left(\mathrm{d}_{+}^{*}+\mathrm{d}\right)_{m+2,-\gamma}^{q}\right)=b^{0}(L)-b^{0}(C)+b^{1}(C) .
$$

The map $\operatorname{Ker}\left(\left(\mathrm{d}_{+}^{*}+\mathrm{d}\right)_{m+2,-\gamma}^{q}\right) \rightarrow H^{3}(C, \mathbb{R}), \chi \mapsto[\chi]$ is surjective, with kernel of dimension $b^{0}(L)-b^{0}(C)+b^{1}(C)-b^{3}(C) \geqslant 0$, which is the dimension of the kernel of $H_{\mathrm{cs}}^{3}(C, \mathbb{R}) \rightarrow H^{3}(C, \mathbb{R})$. So we can think of $\operatorname{Ker}\left(\left(\mathrm{d}_{+}^{*}+\mathrm{d}\right)_{m+2,-\gamma}^{q}\right)$ as a space of closed and coclosed 3 -forms filling out all of $H_{\mathrm{cs}}^{3}(C, \mathbb{R})$ and $H^{3}(C, \mathbb{R})$. But we will not need these facts, so we shall not prove them.

\subsection{Conditions on the rate $\gamma$ for $\mathrm{d}_{+}+\mathrm{d}^{*}$ to be Fredholm}

Finally we determine the set $\mathcal{D}_{\left(\mathrm{d}_{+}+\mathrm{d}^{*}\right)_{0}}$ for the cylindrical operator $\left(\mathrm{d}_{+}+\mathrm{d}^{*}\right)_{0}$ on $L \times \mathbb{R}$, which by Theorem 3.4 gives the set of $\gamma$ for which (20) is not Fredholm.

Proposition 3.11 Let $(C, g)$ be an oriented, asymptotically cylindrical Riemannian 4-manifold asymptotic to $\left(L \times \mathbb{R}, g_{0}\right)$, let $p>1, l \geqslant 0$ and $\gamma \in \mathbb{R}$, and define $\left(\mathrm{d}_{+}+\mathrm{d}^{*}\right)_{l+2, \gamma}^{p}$ as in (20). Then $\left(\mathrm{d}_{+}+\mathrm{d}^{*}\right)_{l+2, \gamma}^{p}$ is not Fredholm if and only if either $\gamma=0$, or $\gamma^{2}$ is a positive eigenvalue of $\Delta=\mathrm{d}^{*} \mathrm{~d}$ on functions on $L$, or $\gamma$ is an eigenvalue of $-* \mathrm{~d}$ on coexact 1 -forms on $L$.

Proof Throughout the proof $*, \mathrm{~d}^{*}$ mean the Hodge star and $\mathrm{d}^{*}$ on $L$, not on $L \times \mathbb{R}$, and $\mathrm{d}_{4}^{*}$ is $\mathrm{d}^{*}$ on $L \times \mathbb{R}$. An element of $C^{\infty}\left(\Lambda_{+}^{2} T^{*}(L \times \mathbb{R}) \otimes_{\mathbb{R}} \mathbb{C}\right)$ invariant under translations in $\mathbb{R}$ may be written uniquely in the form $\chi \wedge \mathrm{d} t+* \chi$ for $\chi \in C^{\infty}\left(T^{*} L \otimes_{\mathbb{R}} \mathbb{C}\right)$. An element of $C^{\infty}\left(\Lambda^{4} T^{*}(L \times \mathbb{R}) \otimes_{\mathbb{R}} \mathbb{C}\right)$ invariant under translations in $\mathbb{R}$ may be written uniquely as $f \mathrm{~d} V_{L} \wedge \mathrm{d} t$ for $f: L \rightarrow \mathbb{C}$ smooth, 
where $\mathrm{d} V_{L}$ is the volume form on $L$. By Definition 3.3 and Theorem 3.4, (20) is not Fredholm if and only if there exist $\delta \in \mathbb{R}$ and $\chi, f$ as above and not both zero, satisfying

$$
\mathrm{d}\left(e^{(\gamma+i \delta) t}(\chi \wedge \mathrm{d} t+* \chi)\right)+\mathrm{d}_{4}^{*}\left(e^{(\gamma+i \delta) t} f \mathrm{~d} V_{L} \wedge \mathrm{d} t\right) \equiv 0 .
$$

Expanding (25) yields

$$
e^{(\gamma+i \delta) t}\left[\mathrm{~d} \chi \wedge \mathrm{d} t+(\gamma+i \delta)(* \chi) \wedge \mathrm{d} t+\mathrm{d}(* \chi)-(* \mathrm{~d} f) \wedge \mathrm{d} t+(\gamma+i \delta) f \mathrm{~d} V_{L}\right] \equiv 0
$$

on $L \times \mathbb{R}$, and separating components with and without $\mathrm{d} t$ gives

$$
\mathrm{d} \chi+(\gamma+i \delta)(* \chi)-(* \mathrm{~d} f) \equiv 0 \quad \text { and } \quad \mathrm{d}(* \chi)+(\gamma+i \delta) f \mathrm{~d} V_{L} \equiv 0,
$$

equations in $2-$ and 3 -forms on $L$ respectively. Applying the Hodge star $*$ on $L$ shows that (25) is equivalent to the two equations

$$
* \mathrm{~d} \chi+(\gamma+i \delta) \chi-\mathrm{d} f \equiv 0 \quad \text { and } \quad \mathrm{d}^{*} \chi-(\gamma+i \delta) f \equiv 0
$$

in 1 -forms and functions on $L$.

Since $\chi$ is a 1 -form on $L$ which is a compact manifold, one can use Hodge decomposition and write $\chi=\chi_{0} \oplus \chi_{1} \oplus \chi_{2}$ where $\chi_{0}$ is a harmonic 1-form, $\chi_{1}=\mathrm{d} f_{1}$ is an exact 1-form, $f_{1} \in C^{\infty}(L)$ and $\chi_{2}=\mathrm{d}^{*} \eta$ is a co-exact 1 -form, $\eta \in C^{\infty}\left(\Lambda^{2} T^{*} L\right)$. Dividing the first equation of (26) into harmonic, exact and coexact components, the system becomes

$$
(\gamma+i \delta) \chi_{0}=0,(\gamma+i \delta) \mathrm{d} f_{1}=\mathrm{d} f,-* \mathrm{~d} \chi_{2}=(\gamma+i \delta) \chi_{2}, \mathrm{~d}^{*} \mathrm{~d} f_{1}=(\gamma+i \delta) f .
$$

The second and fourth equations of (27) give $\mathrm{d}^{*} \mathrm{~d} f=(\gamma+i \delta)^{2} f$, and substituting the third equation into itself gives $\mathrm{d}^{*} \mathrm{~d} \chi_{2}=* \mathrm{~d} * \mathrm{~d} \chi_{2}=(\gamma+i \delta)^{2} \chi_{2}$. But $\mathrm{d}^{*} \chi_{2}=0$ by definition. Thus $\chi_{0}, \chi_{1}, \chi_{2}, f$ satisfy the equations

$$
\begin{aligned}
(\gamma+i \delta) \chi_{0} & =0, & (\gamma+i \delta) \chi_{1} & =\mathrm{d} f, \\
\left(\mathrm{~d}^{*} \mathrm{~d}+\mathrm{dd}^{*}\right) \chi_{2} & =(\gamma+i \delta)^{2} \chi_{2}, & \mathrm{~d}^{*} \mathrm{~d} f & =(\gamma+i \delta)^{2} f .
\end{aligned}
$$

When $\gamma=\delta=0, \chi=0$ and $f \equiv 1$ are a solution, so (20) is not Fredholm. So suppose $\gamma+i \delta \neq 0$. Then $\chi_{0}=0$, and $\chi_{1}=0$ if $f=0$ by (28), so as $\chi, f$ are not both zero either $f \neq 0$ or $\chi_{2} \neq 0$. If $f \neq 0$ then $(\gamma+i \delta)^{2}$ is a nonzero eigenvalue of $\Delta=\mathrm{d}^{*} \mathrm{~d}$ on functions by the last equation of (28), so $\delta=0$ as such eigenvalues are positive, and $\gamma^{2}$ is a positive eigenvalue of $\Delta$ as we want. Conversely, if $\Delta f=\gamma^{2} f$ for nonzero $\gamma, f$ then $\chi=\gamma^{-1} \mathrm{~d} f$ satisfies the equations, so (20) is not Fredholm.

If $\chi_{2} \neq 0$ then $(\gamma+i \delta)^{2}$ is a nonzero eigenvalue of $\Delta=\mathrm{d}^{*} \mathrm{~d}+\mathrm{dd}^{*}$ on coexact 1 -forms by the third equation of (28), so $\delta=0$ as above. The third equation of (27) then shows that $\gamma$ is an eigenvalue of $-* d$ on coexact 1 -forms on $L$. Conversely, taking $\chi_{2}$ to be an eigenvector of $-* d$ on coexact 1 -forms with eigenvalue $\gamma$ and $\chi_{0}=\chi_{1}=f=0$ solves the equations, so (20) is not Fredholm. 


\section{Proof of Theorem 1.1}

We now prove Theorem 1.1] Let $(M, \varphi, g)$ be an asymptotically cylindrical $G_{2}$-manifold asymptotic to $X \times(R, \infty), R>0$, with decay rate $\alpha<0$. Let $C$ be an asymptotically cylindrical coassociative 4 -fold in $X$ asymptotic to $L \times\left(R^{\prime}, \infty\right)$ for $R^{\prime}>R$ with decay rate $\beta$ for $\alpha \leqslant \beta<0$. Write $g_{C}=\left.g\right|_{C}$ for the metric on $C, g_{X}$ for the Calabi-Yau metric on $X$, and $g_{L}=\left.g_{X}\right|_{L}$ for the metric on $L$. Then $\left(C, g_{C}\right)$ is an asymptotically cylindrical Riemannian $4-$ manifold, with rate $\beta$.

Suppose $\gamma$ satisfies $\beta<\gamma<0$, and $\left(0, \gamma^{2}\right]$ contains no eigenvalues of the Laplacian $\Delta_{L}$ on functions on $L$, and $[\gamma, 0)$ contains no eigenvalues of the operator $-* \mathrm{~d}$ on coexact 1 -forms on $L$. Let $p>4$ and $l \geqslant 1$, and define

$$
\left(\mathrm{d}_{+}+\mathrm{d}^{*}\right)_{l+2, \gamma}^{p}: L_{l+2, \gamma}^{p}\left(\Lambda_{+}^{2} T^{*} C\right) \oplus L_{l+2, \gamma}^{p}\left(\Lambda^{4} T^{*} C\right) \longrightarrow L_{l+1, \gamma}^{p}\left(\Lambda^{3} T^{*} C\right)
$$

as in Section 3.3. Then Proposition 3.11 and the conditions on $\gamma$ imply that $[\gamma, 0) \cap \mathcal{D}_{\left(\mathrm{d}_{+}+\mathrm{d}^{*}\right)_{0}}=\emptyset$. Hence $\gamma \notin \mathcal{D}_{\left(\mathrm{d}_{+}+\mathrm{d}^{*}\right)_{0}}$, so that $\left(\mathrm{d}_{+}+\mathrm{d}^{*}\right)_{l+2, \gamma}^{p}$ is Fredholm by Theorem 3.4. Also, Theorem 3.10 applies to $\left(\mathrm{d}_{+}+\mathrm{d}^{*}\right)_{l+2, \gamma}^{p}$.

Let $\nu_{L}$ be the normal bundle of $L$ in $X$, regarded as the orthogonal subbundle to $T L$ in $\left.T X\right|_{L}$, and $\exp _{L}: \nu_{L} \rightarrow X$ the exponential map. For $r>0$, write $B_{r}\left(\nu_{L}\right)$ for the subbundle of $\nu_{L}$ with fibre at $x$ the open ball about 0 in $\left.\nu_{L}\right|_{x}$ with radius $r$. Then for small $\epsilon>0$, there is a tubular neighbourhood $T_{L}$ of $L$ in $X$ such that $\exp _{L}: B_{2 \epsilon}\left(\nu_{L}\right) \rightarrow T_{L}$ is a diffeomorphism. Also, $\nu_{L} \times \mathbb{R} \rightarrow L \times \mathbb{R}$ is the normal bundle to $L \times \mathbb{R}$ in $X \times \mathbb{R}$ with exponential map $\exp _{L} \times$ id: $\nu_{L} \times \mathbb{R} \rightarrow$ $X \times \mathbb{R}$. Then $T_{L} \times \mathbb{R}$ is a tubular neighborhood of $L \times \mathbb{R}$ in $X \times \mathbb{R}$, and $\exp _{L} \times \mathrm{id}: B_{2 \epsilon}\left(\nu_{L}\right) \times \mathbb{R} \rightarrow T_{L} \times \mathbb{R}$ is a diffeomorphism.

Let $K, R, \Psi: X \times(R, \infty) \rightarrow M \backslash K$, and $K^{\prime}, R^{\prime}>R, \Phi: L \times\left(R^{\prime}, \infty\right) \rightarrow C \backslash K^{\prime}$, and the normal vector field $v$ on $L \times\left(R^{\prime}, \infty\right)$ be as in Section 2.3] so that (3) commutes. Then $v$ is a section of $\nu_{L} \times\left(R^{\prime}, \infty\right) \rightarrow L \times\left(R^{\prime}, \infty\right)$, decaying at rate $O\left(e^{\beta t}\right)$. Therefore making $K^{\prime}$ and $R^{\prime}$ larger if necessary, we can suppose the graph of $v$ lies in $B_{\epsilon}\left(\nu_{L}\right) \times\left(R^{\prime}, \infty\right)$.

Write $\pi: B_{\epsilon}\left(\nu_{L}\right) \times\left(R^{\prime}, \infty\right) \rightarrow L \times\left(R^{\prime}, \infty\right)$ for the natural projection. Define

$$
\Xi: B_{\epsilon}\left(\nu_{L}\right) \times\left(R^{\prime}, \infty\right) \rightarrow M \quad \text { by } \quad \Xi: w \mapsto \Psi\left[\left(\exp _{L} \times \mathrm{id}\right)\left(\left.v\right|_{\pi(w)}+w\right)\right] .
$$

Here $w$ is a point in $B_{\epsilon}\left(\nu_{L}\right) \times\left(R^{\prime}, \infty\right)$, in the fibre over $\pi(w) \in L \times\left(R^{\prime}, \infty\right)$. Thus $\left.v\right|_{\pi(w)}$ is a point in the same fibre, which is a ball of radius $\epsilon$ in a vector space.

Hence $\left.v\right|_{\pi(w)}+w$ lies in the open ball of radius $2 \epsilon$ in the same vector space, that is, in the fibre of $B_{2 \epsilon}\left(\nu_{L}\right) \times\left(R^{\prime}, \infty\right)$ over $\pi(w)$. Therefore $\left(\exp _{L} \times \mathrm{id}\right)\left(\left.v\right|_{\pi(w)}+w\right)$ 
is well-defined and lies in $T_{L} \times\left(R^{\prime}, \infty\right) \subset X \times\left(R^{\prime}, \infty\right)$, and $\Xi(w)$ is well-defined. Since $\exp _{L} \times$ id $: B_{2 \epsilon}\left(\nu_{L}\right) \times \mathbb{R} \rightarrow T_{L} \times \mathbb{R}$ and $\Psi: X \times(R, \infty) \rightarrow M \backslash K$ are diffeomorphisms, we see that $\Xi$ is a diffeomorphism with its image.

Identify $L \times\left(R^{\prime}, \infty\right)$ with the zero section in $B_{\epsilon}\left(\nu_{L}\right) \times\left(R^{\prime}, \infty\right)$. Then as (3) commutes we see that $\left.\Xi\right|_{L \times\left(R^{\prime}, \infty\right)} \equiv \Phi$. Let $\nu_{C}$ be the normal bundle of $C$ in $M$, which we regard not as the orthogonal subbundle to $T C$ in $\left.T M\right|_{C}$, but rather as the quotient bundle $\left.T M\right|_{C} / T C$. Define an isomorphism $\xi$ between the vector bundles $\nu_{L} \times\left(R^{\prime}, \infty\right)$ and $\Phi^{*}\left(\nu_{C}\right)$ over $L \times\left(R^{\prime}, \infty\right)$ as follows.

As $\Xi$ is a diffeomorphism with its image, $\mathrm{d} \Xi: T\left(B_{\epsilon}\left(\nu_{L}\right) \times\left(R^{\prime}, \infty\right)\right) \rightarrow \Xi^{*}(T M)$ is an isomorphism. Restricting this to the zero section $L \times\left(R^{\prime}, \infty\right)$ and noting that $\left.\Xi\right|_{L \times\left(R^{\prime}, \infty\right)} \equiv \Phi$, we see that

$$
\left.\mathrm{d} \Xi\right|_{L \times\left(R^{\prime}, \infty\right)}:\left.T\left(B_{\epsilon}\left(\nu_{L}\right) \times\left(R^{\prime}, \infty\right)\right)\right|_{L \times\left(R^{\prime}, \infty\right)} \longrightarrow \Phi^{*}(T M)
$$

is an isomorphism. As $\Phi: L \times\left(R^{\prime}, \infty\right) \rightarrow C \backslash K^{\prime}$ is a diffeomorphism,

$$
\mathrm{d} \Phi: T\left(L \times\left(R^{\prime}, \infty\right)\right) \longrightarrow \Phi^{*}(T N)
$$

is an isomorphism. But (32) is the restriction of (31) to a vector subbundle. Quotienting (31) by (32) gives an isomorphism

$$
\begin{aligned}
\xi=\left.\mathrm{d} \Xi\right|_{L \times\left(R^{\prime}, \infty\right)}: \nu_{L} & \times\left(R^{\prime}, \infty\right) \cong \frac{\left.T\left(B_{\epsilon}\left(\nu_{L}\right) \times\left(R^{\prime}, \infty\right)\right)\right|_{L \times\left(R^{\prime}, \infty\right)}}{T\left(L \times\left(R^{\prime}, \infty\right)\right)} \\
& \longrightarrow \Phi^{*}(T M) / \Phi^{*}(T N) \cong \Phi^{*}(T M / T N)=\Phi^{*}\left(\nu_{C}\right) .
\end{aligned}
$$

Now choose a small $\epsilon^{\prime}>0$, a tubular neighborhood $T_{C}$ of $C$ in $M$, and a diffeomorphism $\Theta: B_{\epsilon^{\prime}}\left(\nu_{C}\right) \rightarrow T_{C}$ satisfying the conditions:

(i) $\left.\Theta\right|_{C} \equiv \operatorname{id}_{C}: C \rightarrow C$, where $C \subset B_{\epsilon^{\prime}}\left(\nu_{C}\right)$ is the zero section.

(ii) By (i), $\left.\mathrm{d} \Theta\right|_{C}:\left.\left.T\left(B_{\epsilon^{\prime}}\left(\nu_{C}\right)\right)\right|_{C} \rightarrow T M\right|_{C}$ is an isomorphism, which restricts to the identity on the subbundles $T C$ of each side. Hence it induces an isomorphism $\left.\mathrm{d} \Theta\right|_{C}:\left.\nu_{C} \cong T\left(B_{\epsilon}\left(\nu_{C}\right)\right)\right|_{C} /\left.T C \rightarrow T M\right|_{C} / T C=\nu_{C}$. This isomorphism is the identity map.

(iii) $\epsilon^{\prime}$ is small enough that $\xi^{*}\left(B_{\epsilon^{\prime}}\left(\nu_{C}\right)\right) \subset B_{\epsilon}\left(\nu_{L}\right) \times\left(R^{\prime}, \infty\right) \subset \nu_{L} \times\left(R^{\prime}, \infty\right)$, and $\Theta \circ \xi \equiv \Xi$ on $\xi^{*}\left(B_{\epsilon^{\prime}}\left(\nu_{C}\right)\right)$.

Notice that (iii) determines $\Theta$ and $T_{C}$ uniquely on $\left.B_{\epsilon^{\prime}}\left(\nu_{C}\right)\right|_{C \backslash K^{\prime}}$, and by construction here it satisfies (i) and (ii). Thus, it remains only to choose $T_{C}$ and $\Theta$ satisfying (i), (ii) over the compact set $K^{\prime} \subset C$, which is possible by standard differential topology.

The point of all this is that we have chosen a local identification $\Theta$ between $\nu_{C}$ and $M$ near $C$ that is compatible in a nice way with the asymptotic identifications $\Phi, \Psi$ of $C, M$ with $L \times \mathbb{R}$ and $X \times \mathbb{R}$. Using $\Theta$, submanifolds $\widetilde{C}$ 
of $M$ close to $C$ are identified with small sections $s$ of $\nu_{C}$, and importantly, the asymptotic convergence of $\widetilde{C}$ to $C$, and so to $L \times \mathbb{R}$, is reflected in the asymptotic convergence of $s$ to 0 .

As in the proof of Theorem 2.5. the map $\left.V \mapsto\left(\left.V \cdot \varphi\right|_{x}\right)\right|_{T_{x} C}$ defines an isomorphism $\nu_{C} \rightarrow \Lambda_{+}^{2} T^{*} C$. (Note that since $\left.\varphi\right|_{C} \equiv 0$, this map is well-defined for $V \in T_{x} M / T_{x} C$, rather than just for $V \in T_{x} M$ orthogonal to $T_{x} C$.) We now identify $\nu_{C}$ with $\Lambda_{+}^{2} T^{*} C$, and regard $\Theta$ as a map $\Theta: B_{\epsilon^{\prime}}\left(\Lambda_{+}^{2} T^{*} C\right) \rightarrow T_{C} \subset M$.

Write $L_{l+2, \gamma}^{p}\left(B_{\epsilon^{\prime}}\left(\Lambda_{+}^{2} T^{*} C\right)\right)$ for the subset of $\zeta_{+}^{2} \in L_{l+2, \gamma}^{p}\left(\Lambda_{+}^{2} T^{*} C\right)$ which are sections of $B_{\epsilon^{\prime}}\left(\Lambda_{+}^{2} T^{*} C\right)$, that is, $\left|\zeta_{+}^{2}\right|<\epsilon^{\prime}$ on $C$. Since $L_{l+1, \gamma}^{p} \hookrightarrow C^{0}$ by Sobolev embedding this is an open condition on $\zeta_{+}^{2}$, so $L_{l+2, \gamma}^{p}\left(B_{\epsilon^{\prime}}\left(\Lambda_{+}^{2} T^{*} C\right)\right)$ is an open subset of the Banach space $L_{l+2, \gamma}^{p}\left(\Lambda_{+}^{2} T^{*} C\right)$.

Define $Q: L_{l+2, \gamma}^{p}\left(B_{\epsilon^{\prime}}\left(\Lambda_{+}^{2} T^{*} C\right)\right) \rightarrow\{3$-forms on $C\}$ by $Q\left(\zeta_{+}^{2}\right)=\left(\Theta \circ \zeta_{+}^{2}\right)^{*}(\varphi)$. That is, we regard the section $\zeta_{+}^{2}$ as a map $C \rightarrow B_{\epsilon^{\prime}}\left(\Lambda_{+}^{2} T^{*} C\right)$, so $\Theta \circ \zeta_{+}^{2}$ is a map $C \rightarrow T_{C} \subset M$, and thus $\left(\Theta \circ \zeta_{+}^{2}\right)^{*}(\varphi)$ is a 3 -form on $C$. The point of this definition is that if $\Gamma_{\zeta_{+}^{2}}$ is the graph of $\zeta_{+}^{2}$ in $B_{\epsilon^{\prime}}\left(\Lambda_{+}^{2} T^{*} C\right)$ and $\widetilde{C}=\Theta\left(\Gamma_{\zeta_{+}^{2}}\right)$ its image in $M$, then $\widetilde{C}$ is coassociative if and only if $\left.\varphi\right|_{\widetilde{C}} \equiv 0$, which holds if and only if $Q\left(\zeta_{+}^{2}\right)=0$. So $Q^{-1}(0)$ parametrizes coassociative 4 -folds $\widetilde{C}$ close to $C$.

We now consider which class of 3 -forms $Q$ maps to.

Proposition $4.1 Q: L_{l+2, \gamma}^{p}\left(B_{\epsilon^{\prime}}\left(\Lambda_{+}^{2} T^{*} C\right)\right) \longrightarrow L_{l+1, \gamma}^{p}\left(\Lambda^{3} T^{*} C\right)$ is a smooth map of Banach manifolds. The linearization of $Q$ at 0 is $\mathrm{d} Q(0): \zeta_{+}^{2} \mapsto \mathrm{d} \zeta_{+}^{2}$.

Proof As in the proof of Theorem [2.5, the functional form of $Q$ is

$$
\left.Q\left(\zeta_{+}^{2}\right)\right|_{x}=F\left(x,\left.\zeta_{+}^{2}\right|_{x},\left.\nabla \zeta_{+}^{2}\right|_{x}\right) \text { for } x \in C,
$$

where $F$ is a smooth function of its arguments. Since $p>4$ and $l \geqslant 1$ we have $L_{l+2, \gamma}^{p}\left(\Lambda_{+}^{2} T^{*} C\right) \hookrightarrow C_{\gamma}^{1}\left(\Lambda_{+}^{2} T^{*} C\right)$ by Sobolev embedding. General arguments then show that locally $Q\left(\zeta_{+}^{2}\right)$ is $L_{l+1}^{p}$.

To show $Q\left(\zeta_{+}^{2}\right)$ lies in $L_{l+1, \gamma}^{p}\left(\Lambda^{3} T^{*} C\right)$, we must know something of the asymptotic behavior of $F$ at infinity. Essentially $Q\left(\zeta_{+}^{2}\right)$ is the restriction to $\Gamma\left(\zeta_{+}^{2}\right)$ of the 3 -form $\Theta^{*}(\varphi)$ on $B_{\epsilon^{\prime}}\left(\Lambda_{+}^{2} T^{*} C\right)$. Using the identifications $\Phi: L^{\prime} \times\left(R^{\prime} \infty\right) \rightarrow$ $C \backslash K^{\prime}$ and $\xi: \nu_{L} \times\left(R^{\prime}, \infty\right) \rightarrow \Phi^{*}\left(\Lambda_{+}^{2} T^{*} C\right)$ over $C \backslash K^{\prime}$, by (iii) above this 3-form becomes $\Xi^{*}(\varphi)$ on $B_{\epsilon}\left(\nu_{L}\right) \times\left(R^{\prime}, \infty\right)$.

But the asymptotic conditions on $\Phi, \Psi$ and $v$ imply that $\Xi^{*}(\varphi)$ is the sum of a translation-invariant 3-form on $B_{\epsilon}\left(\nu_{L}\right) \times \mathbb{R}$, the pullback of the cylindrical $G_{2}$ 
form $\varphi_{0}$ on $X \times \mathbb{R}$, and an error term which decays at rate $O\left(e^{\beta t}\right)$, with all its derivatives. As $\beta<\gamma<0$, it is not difficult to see from this that $Q$ maps to $L_{l+1, \gamma}^{p}\left(\Lambda^{3} T^{*} C\right)$. Smoothness of $Q$ holds by general principles. Finally, the linearization of $Q$ is $\mathrm{d}$, by the calculation of McLean alluded to in Theorem 2.5. As the calculation is local, it does not matter that we are on a noncompact manifold $C$.

Next we show that the image of $Q$ consists of exact 3 -forms. Notice that we lose one degree of differentiability: although the image of $Q$ lies in $L_{l+1, \gamma}^{p}\left(\Lambda^{3} T^{*} C\right)$, we claim only that it lies in the exact 3 -forms in $L_{l, \gamma}^{p}\left(\Lambda^{3} T^{*} C\right)$.

\section{Proposition 4.2}

$$
Q\left(L_{l+2, \gamma}^{p}\left(B_{\epsilon^{\prime}}\left(\Lambda_{+}^{2} T^{*} C\right)\right)\right) \subseteq \mathrm{d}\left(L_{l+1, \gamma}^{p}\left(\Lambda^{2} T^{*} C\right)\right) \subset L_{l, \gamma}^{p}\left(\Lambda^{3} T^{*} C\right) .
$$

Proof Consider the restriction of the 3 -form $\varphi$ to the tubular neighborhood $T_{C}$ of $C$. As $\varphi$ is closed, and $T_{C}$ retracts onto $C$, and $\left.\varphi\right|_{C} \equiv 0$, we see that $\left.\varphi\right|_{T_{C}}$ is exact. Thus we may write $\left.\varphi\right|_{T_{C}}=\mathrm{d} \theta$ for $\theta \in C^{\infty}\left(\Lambda^{2} T^{*} T_{C}\right)$. Since $\left.\varphi\right|_{C} \equiv 0$ we may choose $\left.\theta\right|_{C} \equiv 0$. Also, as $\varphi$ is asymptotic to $O\left(e^{\beta t}\right)$ with all its derivatives to a translation-invariant 3 -form $\varphi_{0}$ on $X \times \mathbb{R}$, we may take $\theta$ to be asymptotic to $O\left(e^{\beta t}\right)$ with all its derivatives to a translation-invariant $2-$ form on $T_{L} \times \mathbb{R}$.

The proof of Proposition 4.1 now shows that the map $\zeta_{+}^{2} \mapsto\left(\Theta \circ \zeta_{+}^{2}\right)^{*}(\theta)$ maps $L_{l+2, \gamma}^{p}\left(B_{\epsilon^{\prime}}\left(\Lambda_{+}^{2} T^{*} C\right)\right) \rightarrow L_{l+1, \gamma}^{p}\left(\Lambda^{2} T^{*} C\right)$. But

$$
Q\left(\zeta_{+}^{2}\right)=\left(\Theta \circ \zeta_{+}^{2}\right)^{*}(\varphi)=\left(\Theta \circ \zeta_{+}^{2}\right)^{*}(\mathrm{~d} \theta)=\mathrm{d}\left[\left(\Theta \circ \zeta_{+}^{2}\right)^{*}(\theta)\right],
$$

so $Q\left(\zeta_{+}^{2}\right) \in \mathrm{d}\left(L_{l+1, \gamma}^{p}\left(\Lambda^{2} T^{*} C\right)\right)$ for $\zeta_{+}^{2} \in L_{l+2, \gamma}^{p}\left(B_{\epsilon^{\prime}}\left(\Lambda_{+}^{2} T^{*} C\right)\right)$.

As in the proof of Theorem 2.5, we augment $Q$ by a space of 4 -forms on $C$ to make it elliptic. Define

$$
\begin{gathered}
P: L_{l+2, \gamma}^{p}\left(B_{\epsilon^{\prime}}\left(\Lambda_{+}^{2} T^{*} C\right)\right) \times L_{l+2, \gamma}^{p}\left(\Lambda^{4} T^{*} C\right) \longrightarrow L_{l+1, \gamma}^{p}\left(\Lambda^{3} T^{*} C\right) \\
\text { by } \quad P\left(\zeta_{+}^{2}, \zeta^{4}\right)=Q\left(\zeta_{+}^{2}\right)+\mathrm{d}^{*} \zeta^{4} .
\end{gathered}
$$

Proposition 4.1 implies that the linearization $\mathrm{d} P(0,0)$ of $P$ at 0 is the Fredholm operator $\left(\mathrm{d}_{+}+\mathrm{d}^{*}\right)_{l+2, \gamma}^{p}$ of (29). Define $\mathcal{C}$ to be the image of $\left(\mathrm{d}_{+}+\mathrm{d}^{*}\right)_{l+2, \gamma}^{p}$. Then $\mathcal{C}$ is a Banach subspace of $L_{l+1, \gamma}^{p}\left(\Lambda^{3} T^{*} C\right)$, since $\left(\mathrm{d}_{+}+\mathrm{d}^{*}\right)_{l+2, \gamma}^{p}$ is Fredholm. We show $P$ maps into $\mathcal{C}$.

Proposition 4.3 $P$ maps $L_{l+2, \gamma}^{p}\left(B_{\epsilon^{\prime}}\left(\Lambda_{+}^{2} T^{*} C\right)\right) \times L_{l+2, \gamma}^{p}\left(\Lambda^{4} T^{*} C\right) \longrightarrow \mathcal{C}$. 
Proof Let $\left(\zeta_{+}^{2}, \zeta^{4}\right) \in L_{l+2, \gamma}^{p}\left(B_{\epsilon^{\prime}}\left(\Lambda_{+}^{2} T^{*} C\right)\right) \times L_{l+2, \gamma}^{p}\left(\Lambda^{4} T^{*} C\right)$ so that $P\left(\zeta_{+}^{2}, \zeta^{4}\right)$ $=Q\left(\zeta_{+}^{2}\right)+\mathrm{d}^{*} \zeta^{4}$ lies in $L_{l+1, \gamma}^{p}\left(\Lambda^{3} T^{*} C\right)$. We must show it lies in $\mathcal{C}$. Since $\gamma \notin \mathcal{D}_{\left(\mathrm{d}_{+}+\mathrm{d}^{*}\right)_{0}}$, from Section 3 this holds if and only if

$$
\left\langle Q\left(\zeta_{+}^{2}\right)+\mathrm{d}^{*} \zeta^{4}, \chi\right\rangle_{L^{2}}=0 \quad \text { for all } \chi \in \operatorname{Ker}\left(\left(\mathrm{d}_{+}^{*}+\mathrm{d}\right)_{m+2,-\gamma}^{q}\right),
$$

where $\frac{1}{p}+\frac{1}{q}=1$ and $m \geqslant 0$.

By Theorem 3.10, $\operatorname{Ker}\left(\left(\mathrm{d}_{+}^{*}+\mathrm{d}\right)_{m+2,-\gamma}^{q}\right)$ consists of closed and coclosed 3-forms $\chi$. By Proposition 4.2 we have $Q\left(\zeta_{+}^{2}\right)=\mathrm{d} \lambda$ for $\lambda \in L_{l+1, \gamma}^{p}\left(\Lambda^{2} T^{*} C\right)$. So

$$
\left\langle Q\left(\zeta_{+}^{2}\right)+\mathrm{d}^{*} \zeta^{4}, \chi\right\rangle_{L^{2}}=\langle\mathrm{d} \lambda, \chi\rangle_{L^{2}}+\left\langle\mathrm{d}^{*} \zeta^{4}, \chi\right\rangle_{L^{2}}=\left\langle\lambda, \mathrm{d}^{*} \chi\right\rangle_{L^{2}}+\left\langle\zeta^{4}, \mathrm{~d} \chi\right\rangle_{L^{2}}=0
$$

for $\chi \in \operatorname{Ker}\left(\left(\mathrm{d}_{+}^{*}+\mathrm{d}\right)_{m+2,-\gamma}^{q}\right)$, as $\chi$ is closed and coclosed, and the inner products and integration by parts are valid because of the matching of rates $\gamma,-\gamma$ and $L^{p}, L^{q}$ with $\frac{1}{p}+\frac{1}{q}=1$. So (34) holds, and $P$ maps into $\mathcal{C}$.

We now apply the Implicit Mapping Theorem for Banach spaces, 7, Theorem 1.2.5].

Theorem 4.4 Let $\mathcal{A}, \mathcal{B}$ and $\mathcal{C}$ be Banach spaces, and $\mathcal{U}, \mathcal{V}$ open neighborhoods of 0 in $\mathcal{A}$ and $\mathcal{B}$. Suppose that the function $P: \mathcal{U} \times \mathcal{V} \rightarrow \mathcal{C}$ is smooth with $P(0,0)=0$, and that $\left.\mathrm{d} P_{(0,0)}\right|_{\mathcal{B}}: \mathcal{B} \rightarrow \mathcal{C}$ is an isomorphism of $\mathcal{B}, \mathcal{C}$ as vector and topological spaces. Then there exists a connected open neighbourhood $\mathcal{U}^{\prime} \subset \mathcal{U}$ of 0 in $\mathcal{A}$ and a unique smooth map $G: \mathcal{U}^{\prime} \rightarrow \mathcal{V}$ such that $G(0)=0$ and $P(x, G(x))=0$ for all $x \in \mathcal{U}^{\prime}$.

Define $\mathcal{A}$ to be $\operatorname{Ker}\left(\left(\mathrm{d}_{+}+\mathrm{d}^{*}\right)_{l+2, \gamma}^{p}\right)$, and $\mathcal{B}$ to be the subspace of $L_{l+2, \gamma}^{p}\left(\Lambda_{+}^{2} T^{*} C\right)$ $\oplus L_{l+2, \gamma}^{p}\left(\Lambda^{4} T^{*} C\right)$ which is $L^{2}$-orthogonal to $\mathcal{A}$. As $\mathcal{A}$ is finite-dimensional and the $L^{2}$ inner product is continuous on $L_{l+2, \gamma}^{p}\left(\Lambda_{+}^{2} T^{*} C\right) \oplus L_{l+2, \gamma}^{p}\left(\Lambda^{4} T^{*} C\right)$, both $\mathcal{A}, \mathcal{B}$ are Banach spaces, and $\mathcal{A} \oplus \mathcal{B}=L_{l+2, \gamma}^{p}\left(\Lambda_{+}^{2} T^{*} C\right) \oplus L_{l+2, \gamma}^{p}\left(\Lambda^{4} T^{*} C\right)$. Choose open neighborhoods $\mathcal{U}, \mathcal{V}$ of 0 in $\mathcal{A}, \mathcal{B}$ such that $\mathcal{U} \times \mathcal{V} \subseteq L_{l+2, \gamma}^{p}\left(B_{\epsilon^{\prime}}\left(\Lambda_{+}^{2} T^{*} C\right)\right) \times$ $L_{l+2, \gamma}^{p}\left(\Lambda^{4} T^{*} C\right)$.

Let $P, \mathcal{C}$ be as above. Then by Propositions 4.1 and 4.3. $P: \mathcal{U} \times \mathcal{V} \rightarrow \mathcal{C}$ is a smooth map of Banach manifolds with $P(0,0)=0$, and linearization $\mathrm{d} P(0,0)=$ $\left(\mathrm{d}_{+}+\mathrm{d}^{*}\right)_{l+2, \gamma}^{p}: \mathcal{A} \oplus \mathcal{B} \rightarrow \mathcal{C}$. By definition $\mathcal{A}$ is the kernel and $\mathcal{C}$ is the image of $\left(\mathrm{d}_{+}+\mathrm{d}^{*}\right)_{l+2, \gamma}^{p}$. Hence $\left.\mathrm{d} P_{(0,0)}\right|_{\mathcal{B}}: \mathcal{B} \rightarrow \mathcal{C}$ is an isomorphism of vector spaces, and as it is a continuous linear map of Banach spaces, it is an isomorphism of topological spaces by the Open Mapping Theorem.

Thus Theorem 4.4 applies, and gives a connected open neighborhood $\mathcal{U}^{\prime}$ of 0 in $\mathcal{U}$, and a smooth map $G: \mathcal{U}^{\prime} \rightarrow \mathcal{V}$ such that $G(0)=0$ and $P(x, G(x)) \equiv 0$. 
Moreover $P^{-1}(0)$ coincides with $\left\{(x, G(x)): x \in \mathcal{U}^{\prime}\right\}$ near $(0,0)$, and so is smooth, finite-dimensional and locally isomorphic to $\mathcal{A}=\operatorname{Ker}\left(\left(\mathrm{d}_{+}+\mathrm{d}^{*}\right)_{l+2, \gamma}^{p}\right)$.

Lemma $4.5 P^{-1}(0)=Q^{-1}(0) \times\{0\}$.

Proof Clearly $Q^{-1}(0) \times\{0\} \subseteq P^{-1}(0)$. Suppose $\left(\zeta_{+}^{2}, \zeta^{4}\right) \in P^{-1}(0)$, so that $Q\left(\zeta_{+}^{2}\right)+\mathrm{d}^{*} \zeta^{4}=0$. We shall show that $\zeta^{4}=0$, so that $Q\left(\zeta_{+}^{2}\right)=0$, and thus $P^{-1}(0) \subseteq Q^{-1}(0) \times\{0\}$. By Proposition 4.2 we have $Q\left(\zeta_{+}^{2}\right)=\mathrm{d} \lambda$ for $\lambda \in L_{l+1, \gamma}^{p}\left(\Lambda^{2} T^{*} C\right)$, so $\mathrm{d} \lambda=-\mathrm{d}^{*} \zeta^{4}$. Hence

$$
\left\|\mathrm{d}^{*} \zeta^{4}\right\|_{L^{2}}^{2}=\left\langle\mathrm{d}^{*} \zeta^{4}, \mathrm{~d}^{*} \zeta^{4}\right\rangle_{L^{2}}=-\left\langle\mathrm{d}^{*} \zeta^{4}, \mathrm{~d} \lambda\right\rangle_{L^{2}}=-\left\langle\zeta^{4}, \mathrm{~d}^{2} \lambda\right\rangle_{L^{2}}=0,
$$

where the inner products and integration by parts are valid as $L_{l+2, \gamma}^{p} \hookrightarrow L_{2}^{2}$. Thus $Q\left(\zeta_{+}^{2}\right)=\mathrm{d}^{*} \zeta^{4}=0$. But $\mathrm{d}^{*} \zeta^{4} \cong \nabla \zeta^{4}$ as $\zeta^{4}$ is a $4-$ form, so $\zeta^{4}$ is constant. Since also $\zeta^{4} \rightarrow 0$ near infinity in $C$, we have $\zeta^{4} \equiv 0$.

Combining this with the previous description of $P^{-1}(0)$ shows that $Q^{-1}(0)$ is smooth, finite-dimensional and locally isomorphic to $\operatorname{Ker}\left(\left(\mathrm{d}_{+}+\mathrm{d}^{*}\right)_{l+2, \gamma}^{p}\right)$. Next we show that $Q^{-1}(0)$ is independent of $l$, and so consists of smooth solutions.

Proposition 4.6 If $\zeta_{+}^{2} \in Q^{-1}(0)$ then $\zeta_{+}^{2} \in L_{m+2, \gamma}^{p}\left(\Lambda_{+}^{2} T^{*} C\right)$ for all $m \geqslant 1$.

Proof Let $m \geqslant 1$ and $\left(\zeta_{+}^{2}, \zeta^{4}\right) \in L_{m+2, \gamma}^{p}\left(B_{\epsilon^{\prime}}\left(\Lambda_{+}^{2} T^{*} C\right)\right) \times L_{m+2, \gamma}^{p}\left(\Lambda^{4} T^{*} C\right)$, so that $P\left(\zeta_{+}^{2}, \zeta^{4}\right)=Q\left(\zeta_{+}^{2}\right)+\mathrm{d}^{*} \zeta^{4}$ lies in $L_{m+1, \gamma}^{p}\left(\Lambda^{3} T^{*} C\right)$. Write $\nabla$ for the Levi-Civita connection of $g_{C}$ on $C$. We shall apply the $\nabla$-Laplacian $\Delta_{C}=$ $g_{C}^{a b} \nabla_{a} \nabla_{b}$ to $P\left(\zeta_{+}^{2}, \zeta^{4}\right)$, using the index notation. Writing $Q$ in terms of $F$ as in Proposition 4.1, we have

$$
\Delta_{C}\left[P\left(\zeta_{+}^{2}, \zeta^{4}\right)\right]=g_{C}^{a b} \nabla_{a} \nabla_{b}\left[F\left(x,\left.\zeta_{+}^{2}\right|_{x},\left.\nabla \zeta_{+}^{2}\right|_{x}\right)\right]+\Delta_{C} \mathrm{~d}^{*} \zeta^{4}
$$

We shall expand (35) in terms of the derivatives of $F$ by the chain rule, using the following notation. Write $F=F(x, y, z)$. Write $\nabla^{x} F$ for the derivative of $F$ 'in the $x$ direction' using $\nabla$. That is, $\left(\nabla^{x} F\right)\left(x^{\prime}, y^{\prime}, z^{\prime}\right)=\nabla\left(\left.F(x, y(x), z(x))\right|_{x^{\prime}}\right.$, where $y(x), z(x)$ satisfy $y\left(x^{\prime}\right)=y^{\prime}, z\left(x^{\prime}\right)=z^{\prime}$ and $(\nabla y)\left(x^{\prime}\right)=(\nabla z)\left(x^{\prime}\right)=0$. Write $\partial^{y} F$ and $\partial^{z} F$ for the partial derivatives of $F$ in the $y, z$ directions. That is, with $x$ held constant the domains $\Lambda_{+}^{2} T_{x}^{*} C$ and $T_{x}^{*} C \otimes \Lambda_{+}^{2} T_{x}^{*} C$ of $y, z$ and the range $\Lambda^{3} T_{x}^{*} C$ of $F$ are vector spaces, so the map $(y, z) \mapsto F(x, y, z)$ is a smooth map between vector spaces, and has well-defined partial derivatives.

Then expanding (35) gives

$$
\Delta_{C}\left[P\left(\zeta_{+}^{2}, \zeta^{4}\right)\right]=L_{\zeta_{+}^{2}}\left(\zeta_{+}^{2}, \zeta^{4}\right)+E\left(x, \zeta_{+}^{2}, \nabla \zeta_{+}^{2}, \nabla^{2} \zeta_{+}^{2}\right)
$$


where $L_{\zeta_{+}^{2}}$ is the linear third-order operator for $0 \leqslant n \leqslant m+1$ given by

$$
\begin{gathered}
L_{\zeta_{+}^{2}}: L_{n+3, \gamma}^{p}\left(\Lambda_{+}^{2} T^{*} C \oplus \Lambda^{4} T^{*} C\right) \longrightarrow L_{n, \gamma}^{p}\left(\Lambda^{3} T^{*} C\right), \\
\left.L_{\zeta_{+}^{2}}\left(\xi_{+}^{2}, \xi^{4}\right)\right|_{x}=\left.\left(\partial^{z} F\right)\left(x,\left.\zeta_{+}^{2}\right|_{x},\left.\nabla \zeta_{+}^{2}\right|_{x}\right) \cdot \Delta_{C} \nabla \xi_{+}^{2}\right|_{x}+\left.\Delta_{C} \mathrm{~d}^{*} \xi^{4}\right|_{x},
\end{gathered}
$$

and $E\left(x, \zeta_{+}^{2}, \nabla \zeta_{+}^{2}, \nabla^{2} \zeta_{+}^{2}\right)$ is the 'error term' given by

$$
\begin{gathered}
E\left(x, \zeta_{+}^{2}, \nabla \zeta_{+}^{2}, \nabla^{2} \zeta_{+}^{2}\right)=\left(\partial^{y} F\right)(x, y, z) \cdot \Delta_{C} \zeta_{+}^{2}+g_{C}^{a b}\left(\nabla_{a}^{x} \nabla_{b}^{x} F\right)(x, y, z) \\
+g_{C}^{a b}\left(\nabla_{a}^{x} \partial^{y} F\right)(x, y, z) \cdot \nabla_{b} \zeta_{+}^{2}+g_{C}^{a b}\left(\nabla_{a}^{x} \partial^{z} F\right)(x, y, z) \cdot \nabla_{b} \nabla \zeta_{+}^{2} \\
+g_{C}^{a b}\left(\partial^{y} \nabla_{b}^{x} F\right)(x, y, z) \cdot \nabla_{a} \zeta_{+}^{2}+g_{C}^{a b}\left(\partial^{y} \partial^{y} F\right)(x, y, z) \cdot\left(\nabla_{a} \zeta_{+}^{2} \otimes \nabla_{b} \zeta_{+}^{2}\right) \\
+g_{C}^{a b}\left(\partial^{y} \partial^{z} F\right)(x, y, z) \cdot\left(\nabla_{a} \zeta_{+}^{2} \otimes \nabla_{b} \nabla \zeta_{+}^{2}\right)+g_{C}^{a b}\left(\partial^{z} \nabla_{b}^{x} F\right)(x, y, z) \cdot \nabla_{a} \nabla \zeta_{+}^{2} \\
+g_{C}^{a b}\left(\partial^{z} \partial^{y} F\right)(x, y, z) \cdot\left(\nabla_{a} \nabla \zeta_{+}^{2} \otimes \nabla_{b} \zeta_{+}^{2}\right) \\
+g_{C}^{a b}\left(\partial^{z} \partial^{z} F\right)(x, y, z) \cdot\left(\nabla_{a} \nabla \zeta_{+}^{2} \otimes \nabla_{b} \nabla \zeta_{+}^{2}\right) .
\end{gathered}
$$

Here in (37) and (38) we have used '. ' to denote various natural bilinear products, and in (38) we write $y=\left.\zeta_{+}^{2}\right|_{x}$ and $z=\left.\nabla \zeta_{+}^{2}\right|_{x}$ in the arguments of $F$.

The point is that (36) splits $\Delta_{C}\left[P\left(\zeta_{+}^{2}, \zeta^{4}\right)\right]$ up into the piece $L_{\zeta_{+}^{2}}\left(\zeta_{+}^{2}, \zeta^{4}\right)$ containing all the third derivative terms of $\zeta_{+}^{2}, \zeta^{4}$, plus a term $E\left(x, \zeta_{+}^{2}, \nabla \zeta_{+}^{2}, \nabla^{2} \zeta_{+}^{2}\right)$ depending only on $\zeta_{+}^{2}$ up to second derivatives. Furthermore, we may write $L_{\zeta_{+}^{2}}\left(\zeta_{+}^{2}, \zeta^{4}\right)$ as a linear operator $L_{\zeta_{+}^{2}}$ applied to $\left(\zeta_{+}^{2}, \zeta^{4}\right)$, where the coefficients of $L_{\zeta_{+}^{2}}$ depend on $\zeta_{+}^{2}$ only up to first derivatives.

The operator $L_{\zeta_{+}^{2}}$ is essentially $\Delta_{C}\left(\mathrm{~d}_{+}+\mathrm{d}^{*}\right)$, and so is a linear third-order elliptic operator. Note that we allow $0 \leqslant n \leqslant m+1$ in (37). The reason for this is that the coefficients of $L_{\zeta_{+}^{2}}$ depend on $\zeta_{+}^{2}, \nabla \zeta_{+}^{2}$, and so lie in $L_{m+1}^{p}$ locally. So the maximum regularity we can expect for $L_{\zeta_{+}^{2}}\left(\xi_{+}^{2}, \xi^{4}\right)$ is $L_{m+1}^{p}$, forcing $n \leqslant m+1$.

The most obvious value for $n$ in (37) is $n=m-1$, as then the domain of $L_{\zeta_{+}^{2}}$ is the space $L_{m+2, \gamma}^{p}$ containing $\left(\zeta_{+}^{2}, \zeta^{4}\right)$. However, our next lemma is an elliptic regularity result for $L_{\zeta_{+}^{2}}$ when $n=m$. This is because we will use it to increase the regularity of $\left(\zeta_{+}^{2}, \zeta^{4}\right)$ from $L_{m+2, \gamma}^{p}$ to $L_{m+3, \gamma}^{p}$ by 'bootstrapping'.

Lemma 4.7 There exists $A>0$ such that if $\left(\xi_{+}^{2}, \xi^{4}\right) \in L_{3, \gamma}^{p}\left(\Lambda_{+}^{2} T^{*} C \oplus \Lambda^{4} T^{*} C\right)$ with $L_{\zeta_{+}^{2}}\left(\xi_{+}^{2}, \xi^{4}\right) \in L_{m, \gamma}^{p}\left(\Lambda^{3} T^{*} C\right)$ then $\left(\xi_{+}^{2}, \xi^{4}\right) \in L_{m+3, \gamma}^{p}\left(\Lambda_{+}^{2} T^{*} C \oplus \Lambda^{4} T^{*} C\right)$, and

$$
\left\|\left(\xi_{+}^{2}, \xi^{4}\right)\right\|_{L_{m+3, \gamma}^{p}} \leqslant A\left(\left\|L_{\zeta_{+}^{2}}\left(\xi_{+}^{2}, \xi^{4}\right)\right\|_{L_{m, \gamma}^{p}}+\left\|\left(\xi_{+}^{2}, \xi^{4}\right)\right\|_{L_{0, \gamma}^{p}}\right) .
$$


Proof If $L_{\zeta_{+}^{2}}$ were a smooth, asymptotically cylindrical elliptic operator the lemma would follow from Lockhart and McOwen [15, Theorem 3.7.2], 16, Equation (2.4)]. We have to deal with the fact that the coefficients of $L_{\zeta_{+}^{2}}$ depend on $\zeta_{+}^{2}, \nabla \zeta_{+}^{2}$, and so are only $L_{m+1}^{p}$ locally rather than smooth. Unfortunately, Lockhart and McOwen's proof [16, p420] is not very informative, saying only that (39) is established 'by standard parametric techniques'.

Local results of this form are proved by Morrey [23, Section 6.2]. Supposing only that the coefficients of the third-order elliptic operator $L_{\zeta_{+}^{2}}$ are $C^{m}$, Morrey [23, Theorem 6.2.5] implies that if $\left(\xi_{+}^{2}, \xi^{4}\right)$ is locally $L_{3}^{p}$ and $L_{\zeta_{+}^{2}}\left(\xi_{+}^{2}, \xi^{4}\right)$ is locally $L_{m}^{p}$ then $\left(\xi_{+}^{2}, \xi^{4}\right)$ is locally $L_{m+3}^{p}$. Also, [23, Theorem 6.2.6] proves a local interior estimate of the form (39), where $A>0$ depends on $m, p$, the domains involved, $C^{m}$ bounds on the coefficients of $L_{\zeta_{+}^{2}}$, and a modulus of continuity for their $m^{\text {th }}$ derivatives.

Such a modulus of continuity is provided by a Hölder $C^{0, \alpha}$ bound for the $m^{\text {th }}$ derivatives, for $\alpha \in(0,1)$. Thus, we can prove local estimates of the form (39) provided we have local $C^{m, \alpha}$ bounds for the coefficients of $L_{\zeta_{+}^{2}}$, which follow from local $C^{m+1, \alpha}$ bounds for $\zeta_{+}^{2}$. Now $\zeta_{+}^{2} \in L_{m+2, \gamma}^{p}\left(\Lambda_{+}^{2} T^{*} C\right)$ and $p>4$, so the Sobolev Embedding Theorem shows that $L_{m+2}^{p}$ embeds in $C^{m+1, \alpha}$ for $\alpha=1-4 / p$. Therefore we do have $C^{m, \alpha}$ control on the coefficients of $L_{\zeta_{+}^{2}}$, and can show that $L_{\zeta_{+}^{2}}$ is asymptotic in a weighted Hölder $C_{\gamma}^{m, \alpha}$ sense to a cylindrical operator. So (39) holds as in [16, and the lemma is proved.

Suppose now that $\zeta_{+}^{2} \in Q^{-1}(0)$. Then for $m=l \geqslant 1$ we have $\left(\zeta_{+}^{2}, 0\right) \in$ $L_{m+2, \gamma}^{p}\left(B_{\epsilon^{\prime}}\left(\Lambda_{+}^{2} T^{*} C\right)\right) \times L_{m+2, \gamma}^{p}\left(\Lambda^{4} T^{*} C\right)$, with $P\left(\zeta_{+}^{2}, 0\right) \equiv 0$. So (36) gives

$$
L_{\zeta_{+}^{2}}\left(\zeta_{+}^{2}, 0\right)=-E\left(x, \zeta_{+}^{2}, \nabla \zeta_{+}^{2}, \nabla^{2} \zeta_{+}^{2}\right)
$$

As $\zeta_{+}^{2} \in L_{m+2, \gamma}^{p}\left(\Lambda_{+}^{2} T^{*} C\right)$ we see from (38) and the asymptotic behavior of $F$ that the right hand side of (40) lies in $L_{m, \gamma}^{p}\left(\Lambda^{3} T^{*} C\right)$. Lemma 4.7 with $\left(\xi_{+}^{2}, \xi^{4}\right)=$ $\left(\zeta_{+}^{2}, 0\right)$ then shows that $\zeta_{+}^{2} \in L_{m+3, \gamma}^{p}\left(\Lambda_{+}^{2} T^{*} C\right)$. So we have increased the regularity of $\zeta_{+}^{2}$ by one derivative. By induction we have $\zeta_{+}^{2} \in L_{m+2, \gamma}^{p}\left(\Lambda_{+}^{2} T^{*} C\right)$ for $m=l, l+1, l+2, \ldots$, and Proposition 4.6 is proved.

Now let $\mathcal{M}_{C}^{\gamma}$ be the moduli space of asymptotically cylindrical coassociative submanifolds in $M$ close to $C$, and asymptotic to $L \times\left(R^{\prime}, \infty\right)$ with decay rate $\gamma$, as in Theorem 1.1. Define a map $S: Q^{-1}(0) \rightarrow\{4$-submanifolds of $M\}$ by $S: \zeta_{+}^{2} \rightarrow \Theta\left(\Gamma_{\zeta_{+}^{2}}\right)$, where $\Gamma_{\zeta_{+}^{2}}$ is the graph of $\zeta_{+}^{2}$ in $B_{\epsilon^{\prime}}\left(\Lambda_{+}^{2} T^{*} C\right)$ as above. 
Proposition 4.8 $S$ is a homeomorphism from $Q^{-1}(0)$ to a neighborhood of $C$ in $\mathcal{M}_{C}^{\gamma}$.

Proof First we must show that $S$ maps $Q^{-1}(0) \rightarrow \mathcal{M}_{C}^{\gamma}$. Let $\zeta_{+}^{2} \in Q^{-1}(0)$, and set $\widetilde{C}=S\left(\zeta_{+}^{2}\right)$. Then $\left(\Theta \circ \zeta_{+}^{2}\right)^{*}(\varphi)=Q\left(\zeta_{+}^{2}\right)=0$, so $\left.\varphi\right|_{\widetilde{C}}=0$ as $\widetilde{C}$ is the image of the map $\Theta \circ \zeta_{+}^{2}: C \rightarrow M$. Hence $\widetilde{C}$ is coassociative. Proposition 4.6 and Sobolev Embedding shows that $\zeta_{+}^{2}$ is smooth, so $\widetilde{C}$ is a smooth submanifold of $M$.

To show $\widetilde{C}$ is asymptotically cylindrical with rate $\gamma$, note that $\Theta \circ \zeta_{+}^{2}: C \rightarrow \widetilde{C}$ is a diffeomorphism. Define $\widetilde{K}^{\prime}=\Theta \circ \zeta_{+}^{2}\left(K^{\prime}\right)$ and $\widetilde{\Phi}: L \times\left(R^{\prime}, \infty\right) \rightarrow \widetilde{C} \backslash \widetilde{K}^{\prime}$ by $\widetilde{\Phi}=\Theta \circ \zeta_{+}^{2} \circ \Phi$. Then $\widetilde{K}^{\prime}$ is compact, and $\widetilde{\Phi}$ is a diffeomorphism. Now $\zeta_{+}^{2}$ is a section of $\Lambda_{+}^{2} T^{*} C \cong \nu_{C}$, so $\Phi^{*}\left(\zeta_{+}^{2}\right)$ is a section of $\Phi^{*}\left(\nu_{C}\right)$ over $L \times\left(R^{\prime}, \infty\right)$. Pulling back by the isomorphism $\xi$ gives a section $\xi^{*} \circ \Phi^{*}\left(\zeta_{+}^{2}\right)$ of the vector bundle $\nu_{L} \times\left(R^{\prime}, \infty\right)$ over $L \times\left(R^{\prime}, \infty\right)$.

Now the normal vector field $v$ on $L \times\left(R^{\prime}, \infty\right)$ making (3) commute is also a section of $\nu_{L} \times\left(R^{\prime}, \infty\right)$. Define $\widetilde{v}=v+\xi^{*} \circ \Phi^{*}\left(\zeta_{+}^{2}\right)$. Then $\widetilde{v}$ is a section of $L \times$ $\left(R^{\prime}, \infty\right)$, and the definition of $\Xi$ in (30) and part (iii) of the definition of $\Theta$ after (33) show that (3) commutes with $C, K^{\prime}, \Phi, v$ replaced by $\widetilde{C}, \widetilde{K}^{\prime}, \widetilde{\Phi}, \widetilde{v}$. Therefore by Definition 2.9, $\widetilde{C}$ is asymptotically cylindrical with rate $\gamma$ if $\left|\nabla^{k} \widetilde{v}\right|=O\left(e^{\gamma t}\right)$ on $L \times\left(R^{\prime}, \infty\right)$ for all $k \geqslant 0$.

As $C$ is asymptotically cylindrical with rate $\beta<\gamma$ we have $\left|\nabla^{k} v\right|=O\left(e^{\beta t}\right)$ for all $k \geqslant 0$. Proposition 4.6 and Sobolev Embedding for weighted spaces, which holds as in Lockhart [15, Theorem 3.10] and Bartnik [2, Theorem 1.2], then imply that $\left|\nabla^{k} \zeta_{+}^{2}\right|=O\left(e^{\gamma \rho}\right)$ on $C$ for all $k \geqslant 0$, where $\rho$ is as in Definition 3.1. Therefore $\nabla^{k}\left(\xi^{*} \circ \Phi^{*}\left(\zeta_{+}^{2}\right)\right)=O\left(e^{\gamma t}\right)$ on $L \times\left(R^{\prime}, \infty\right)$, as $\Phi^{*}(\rho) \equiv t$, and $\xi, \Phi$ are asymptotically cylindrical. As $\beta<\gamma$ this gives $\left|\nabla^{k} \widetilde{v}\right|=O\left(e^{\gamma t}\right)$ for all $k \geqslant 0$, and $\widetilde{C}$ is asymptotically cylindrical with weight $\gamma$. Hence $S$ maps $Q^{-1}(0) \rightarrow$ $\mathcal{M}_{C}^{\gamma}$.

Next we reverse the argument. Suppose $\widetilde{C}$ is close to $C$ in $\mathcal{M}_{C}^{\gamma}$. As $\widetilde{C}, C$ are $C^{1}$ close there exists a unique smooth section $\zeta_{+}^{2}$ of $B_{\epsilon^{\prime}}\left(\Lambda_{+}^{2} T^{*} C\right)$ such that $\Theta \circ \zeta_{+}^{2}: C \rightarrow \widetilde{C}$ is a diffeomorphism. Since $\left.\varphi\right|_{\widetilde{C}} \equiv 0$ we have $Q\left(\zeta_{+}^{2}\right)=$ $\left(\Theta \circ \zeta_{+}^{2}\right)^{*}(\varphi)=0$. Let $\widetilde{C}$ have data $\widetilde{K}^{\prime}, \widetilde{\Phi}, \widetilde{v}$ as in Definition 2.9. The argument above shows that $\widetilde{v}=v+\xi^{*} \circ \Phi^{*}\left(\zeta_{+}^{2}\right)$. But $\left|\nabla^{k} v\right|=O\left(e^{\gamma t}\right)$ and $\left|\nabla^{k} \widetilde{v}\right|=O\left(e^{\gamma t}\right)$ for all $k \geqslant 0$. Subtracting implies that $\left|\nabla^{k} \zeta_{+}^{2}\right|=O\left(e^{\gamma \rho}\right)$ on $C$ for all $k \geqslant 0$.

We need to show that $\zeta_{+}^{2} \in L_{l+2, \gamma}^{p}\left(B_{\epsilon^{\prime}}\left(\Lambda_{+}^{2} T^{*} C\right)\right)$. The estimate $\left|\nabla^{k} \zeta_{+}^{2}\right|=$ $O\left(e^{\gamma \rho}\right)$ for $k \geqslant 0$ does not prove this, however it does imply that $\zeta_{+}^{2}$ lies in 
$L_{l+2, \gamma^{\prime}}^{p}\left(B_{\epsilon^{\prime}}\left(\Lambda_{+}^{2} T^{*} C\right)\right)$ for any $\gamma^{\prime}>\gamma$. Hence $\zeta_{+}^{2}$ lies in $Q^{\prime-1}(0)$, where $Q^{\prime}$ is $Q$ defined with rate $\gamma^{\prime}$.

Choose $\gamma^{\prime}>\gamma$ such that $\left[\gamma, \gamma^{\prime}\right] \cap \mathcal{D}_{\left(\mathrm{d}_{+}+\mathrm{d}^{*}\right)_{0}}=\emptyset$. Then $Q^{-1}(0) \subseteq Q^{\prime-1}(0)$, and from above $Q^{-1}(0), Q^{\prime-1}(0)$ are smooth, finite-dimensional and locally isomorphic to $\operatorname{Ker}\left(\left(\mathrm{d}_{+}+\mathrm{d}^{*}\right)_{l+2, \gamma}^{p}\right), \operatorname{Ker}\left(\left(\mathrm{d}_{+}+\mathrm{d}^{*}\right)_{l+2, \gamma^{\prime}}^{p}\right)$ respectively. But as $\left[\gamma, \gamma^{\prime}\right] \cap \mathcal{D}_{\left(\mathrm{d}_{+}+\mathrm{d}^{*}\right)_{0}}=\emptyset$ these kernels are equal, so $Q^{-1}(0)$ and $Q^{\prime-1}(0)$ coincide near 0 . Thus if $\widetilde{C}$ is close enough to $C$ in $\mathcal{M}_{C}^{\gamma}$ then $\zeta_{+}^{2}$ lies in $Q^{-1}(0)$, as we want.

To show $S$ is a homeomorphism requires us to specify the topology on $\mathcal{M}_{C}^{\gamma}$, which we have not done. The natural way to define a topology on a space of submanifolds is to identify submanifolds $\widetilde{C}$ near $C$ with sections of the normal bundle $\nu_{C}$ to $C$, and induce the topology from some Banach norm on a space of sections of $\nu_{C}$. In our case, this just means that the topology on $\mathcal{M}_{C}^{\gamma}$ is induced from some choice of Banach norm on sections $\zeta_{+}^{2}$ of $\Lambda_{+}^{2} T^{*} C$, say the $C_{\gamma}^{1}$ topology.

But since $Q^{-1}(0)$ with its $L_{l+2, \gamma}^{p}$ topology is locally homeomorphic to the finitedimensional vector space $\operatorname{Ker}\left(\left(\mathrm{d}_{+}+\mathrm{d}^{*}\right)_{l+2, \gamma}^{p}\right)$, any choice of topology on sections $\zeta_{+}^{2}$ gives the same topology on $\mathcal{M}_{C}^{\gamma}$, as all Banach norms give the same topology on a finite-dimensional space. So $S$ is a local homeomorphism.

As from above $Q^{-1}(0)$ is smooth, finite-dimensional and locally isomorphic to $\operatorname{Ker}\left(\left(\mathrm{d}_{+}+\mathrm{d}^{*}\right)_{l+2, \gamma}^{p}\right)$, Theorem 3.10 and Proposition 4.8 now prove Theorem 1.1

Remark 4.9 In a forthcoming paper, we study the deformation space of asymptotically cylindrical coassociative submanifolds with moving boundary. Similarly to the setting here, we work with an asymptotically cylindrical $G_{2}-$ manifold $M$ with a Calabi-Yau boundary $X$ at infinity. We also assume that the boundary of the coassociative submanifold at infinity is a special Lagrangian submanifold of $X$ and is allowed to move. The details of this construction will appear in [26].

Acknowledgements Part of this work was done when the second author was visiting Oxford University during Fall 2003. Many thanks to the AWM for their grant support which made this visit possible. We also would like to thank Conan Leung for bringing this problem to our attention. The first author would like to thank Jason Lotay for useful conversations. 


\section{References}

[1] S Akbulut, S Salur, Calibrated Manifolds and Gauge Theory, arXiv:math.GT/0402368

[2] R Bartnik, The Mass of an Asymptotically Flat Manifold, Comm. Pure Appl. Math. 39 (1986) 661-693 MathReview

[3] R L Bryant, S M Salamon, On the Construction of some Complete Metrics with Exceptional Holonomy, Duke Math. J. 58 (1989) 829-850 MathReview

[4] J Cheeger, D Gromoll, The splitting theorem for manifolds of nonnegative Ricci curvature, J. Differential Geometry 6 (1971/72) 119-128 MathReview

[5] F R Harvey, H B Lawson, Calibrated Geometries, Acta. Math. 148 (1982) 47-157 MathReview

[6] M Ionel, S Karigiannis, M Min-Oo, Bundle Constructions of Calibrated Submanifolds in $\mathbb{R}^{7}$ and $\mathbb{R}^{8}$, arXiv:math.DG/0408005

[7] D D Joyce, Compact Manifolds with Special Holonomy, Oxford University Press, Oxford, UK (2000) MathReview

[8] D D Joyce, Lectures on Calabi-Yau and special Lagrangian geometry, arXiv:math.DG/0108088

[9] D D Joyce, Special Lagrangian submanifolds with isolated conical singularities I: Regularity, Ann. Global Anal. Geom. 25 (2004) 201-251 MathReview

[10] D D Joyce, Special Lagrangian submanifolds with isolated conical singularities II: Moduli spaces, Ann. Global Anal. Geom. 25 (2004), 301-352 MathReview

[11] A G Kovalev, Twisted connected sums and special Riemannian holonomy, J. Reine Angew. Math. 565 (2003) 125-160 MathReview

[12] C L Leung, Lectures on $G_{2}$ geometry, IPAM Lecture Notes (2003)

[13] C L Leung, Topological Quantum Field Theory for Calabi-Yau threefolds and $G_{2}-$ manifolds, from: "Surveys in differential geometry, Vol. VIII (Boston, MA, 2002)", 257-267, Int. Press, Somerville, MA (2003) 257-267 MathReview

[14] C L Leung, X Wang, Intersection theory of coassociative submanifolds in $G_{2}$ manifolds and Seiberg-Witten Invariants, arXiv:math.DG/0401419

[15] R B Lockhart, Fredholm, Hodge and Liouville Theorems on noncompact manifolds, Trans. Amer. Math. Soc. 301 (1987) 1-35 MathReview

[16] R B Lockhart, R C McOwen, Elliptic differential operators on noncompact manifolds, Ann. Scuola Norm. Sup. Pisa, Classe di Scienze 12 (1987) 409-447 MathReview

[17] J Lotay, Constructing Associative 3-folds by Evolution Equations, e-print, arXiv:math.DG/0401123

[18] J Lotay, 2-Ruled Calibrated 4-folds in $\mathbb{R}^{7}$ and $\mathbb{R}^{8}$, arXiv:math.DG/0401125 
[19] S P Marshall, Deformations of special Lagrangian submanifolds, DPhil thesis, University of Oxford (2002)

[20] R Melrose, The Atiyah-Patodi-Singer Index Theorem, Research Notes in Mathematics, 4, AK Peters, Wellesley, MA, USA (1994) MathReview

[21] R Melrose, Spectral and Scattering Theory for the Laplacian on Asymptotically Euclidean Spaces, from: "Spectral and Scattering Theory" (M Ikawa, editor), Lecture Notes in Pure and Applied Mathematics 161, Marcel Dekker Inc. (1994) 85-130 MathReview

[22] R C McLean, Deformations of calibrated submanifolds, Comm. Anal. Geom. 6 (1998) 705-747 MathReview

[23] C B Morrey Jr, Multiple Integrals in the Calculus of Variations, Grund. der math. Wiss. 130, Springer-Verlag, Berlin, Germany (1966) MathReview

[24] T Pacini, Deformations of Asymptotically Conical Special Lagrangian Submanifolds, Pacific J. Math. 215 (2004) 151-181 MathReview

[25] S Salur, Asymptotically Cylindrical Ricci-Flat Manifolds, to appear in Proc. Amer. Math. Soc. arXiv:math.DG/0410063

[26] S Salur, Deformations of Asymptotically Cylindrical Coassociative Submanifolds with Moving Boundary, in preparation (2005) 